\title{
TITLE: Magnetically-r
}

esponsive, multifunctional drug delivery nanoparticles for elastic matrix regenerative repair

Authors: Balakrishnan Sivaraman ${ }^{1}$, Ganesh Swaminathan ${ }^{1,2}$, Lee Moore ${ }^{1}$, Jonathan Fox ${ }^{1}$, Dhruv Seshadri ${ }^{1}$, Shataakshi Dahal ${ }^{1}$, Ivan Stoilov ${ }^{3}$, Maciej Zborowski ${ }^{1}$, Robert Mecham ${ }^{3}$, Anand Ramamurthi ${ }^{1,3 *}$

Affiliation: ${ }^{1}$ Department of Biomedical Engineering, Cleveland Clinic, 9500 Euclid Avenue, ND 20, Cleveland, OH 44195, USA; ${ }^{2}$ Department of Biology, University of Akron, Akron, OH; ${ }^{3}$ Department of Cell Biology and Physiology, Washington University, St. Louis, MO, ${ }^{3}$ Department of Molecular Medicine, Cleveland Clinic Lerner College of Medicine of Case Western Reserve University, Cleveland, $\mathrm{OH}$.

* Corresponding author: Anand Ramamurthi; e-mail: ramamua@ccf.org 


\section{Abstract}

Arresting or regressing growth of abdominal aortic aneurysms (AAAs), localized expansions of the abdominal aorta are contingent on inhibiting chronically overexpressed matrix metalloproteases (MMPs)-2 and -9 that disrupt elastic matrix within the aortic wall, concurrent with providing a stimulus to augmenting inherently poor auto-regeneration of these matrix structures. In a recent study we demonstrated that localized, controlled and sustained delivery of doxycycline (DOX; a tetracycline-based antibiotic) from poly(lactic-co-glycolic acid) nanoparticles (PLGA NPs), enhances elastic matrix deposition and MMP-inhibition at a fraction of the therapeutically effective oral dose. The surface functionalization of these NPs with cationic amphiphiles, which enhances their arterial uptake, was also shown to have pro-matrix regenerative and anti-MMP effects independent of the DOX. Based on the hypothesis that the incorporation of superparamagnetic iron oxide NPs (SPIONs) within these PLGA NPs would enhance their targetability to the AAA site under an applied external magnetic field, we sought to evaluate the functional effects of NPs co-encapsulating DOX and SPIONs (DOX-SPION NPs) on elastic matrix regeneration and MMP synthesis/activity in vitro within aneurysmal smooth muscle cell (EaRASMC) cultures. The DOX-SPION NPs were mobile under an applied external magnetic field, while enhancing elastic matrix deposition 1.5-2 fold and significantly inhibiting MMP-2 synthesis and MMP-2 and -9 activities, compared to NP-untreated control cultures. These results illustrate that the multifunctional benefits of NPs are maintained following SPION co-incorporation. Additionally, preliminary studies carried out demonstrated enhanced 
targetability of SPION-loaded NPs within proteolytically-disrupted porcine carotid arteries $e x$ vivo, under the influence of an applied external magnetic field. Thus, this dual-agent loaded NP system proffers a potential non-surgical option for treating small growing AAAs, via controlled and sustained drug release from multifunctional, targetable nanocarriers.

Keywords: Nanoparticles; Elastic Matrix; Regenerative Matrix Repair; Drug Delivery; Aortic Aneurysms; Smooth Muscle Cells; Magnetic Targeting 


\section{INTRODUCTION}

Abdominal aortic aneurysms (AAAs) are localized, rupture-prone expansions of the infrarenal abdominal aorta resulting from progressive breakdown of wall elastic matrix by chronically overexpressed matrix metalloproteases (MMPs), specifically MMPs 2 and 9 [1]. AAAs afflict between 1-9\% of men and 1-2 \% of women in developed nations [2], and is the cause of $1-3 \%$ of all deaths in men above the age of 65 [3]. Greater than $90 \%$ of diagnosed AAAs tend to small with a maximal diameter less than a critical size of $5.5 \mathrm{~cm}$, above which rupture risk greatly increases [4]. These AAAs typically grow slowly ( 10\% per year) to the critical size $[5,6]$ over $\geq 5$ years during which period, they are only managed through periodic ultrasound-based growth monitoring. Surgical or minimally invasive interventions are applicable only to larger AAAs and provide no benefit to treating sub-critically sized AAAs. Non-surgical approaches to arrest or regress small AAA growth during the monitoring period are thus warranted.

Restoring elastic matrix homeostasis in the AAA wall is essential to achieving AAA growth arrest or regression to a healthy vessel state. This mandates a) inhibiting chronic proteolysis by elastolytic MMPs-2 and -9 overexpressed by inflammatory cells $[7,8]$ and activated smooth muscle cells (SMCs) within the AAA wall $[2,9]$, and b) augmenting elastic matrix regeneration, which adult $[10,11]$ and aneurysmal [12] SMCs are naturally incapable of achieving. Recent studies in human patients $[5,6,13-18]$ and in animal models $[13,16,19,20]$ suggest that oral dosing of doxycycline (DOX), a modified tetracycline, is useful to inhibit transcription [21-23] and activity $[24,25]$ of elastolytic MMPs in the AAA wall and thus slow their growth. However, drawbacks of oral DOX dosing include systemic side effects [13], and bodywide inhibition of MMPs which can adversely affect healthy tissue remodeling. In addition, elastic matrix synthesis 
inhibitory effects of the drug at the therapeutically useful oral doses (equivalent to tissue level concentrations of $16-54 \mu \mathrm{g} / \mathrm{mL}$ ) [26] is also a concern. The latter aspect has been confirmed in in vitro cultures of vascular SMCs as well $[26,27]$. On this basis, modalities for AAA tissuelocalized, predictable, and and sustained delivery of DOX must be pursued.

Recent studies have demonstrated feasibility of peri-aortic DOX delivery at $1 / 100^{\text {th }}$ of the oral dose from implanted micropumps [28, 29], and shown it to be effective in suppressing AAA formation [28]. While promising, long-term pump implantation is concerning and clinically unacceptable. Accordingly, we recently investigated in aneurysmal SMC cultures, the utility of controlled and sustained DOX delivery from poly(lactic-co-glycolic acid) nanoparticles (PLGA NPs) in attenuating MMPs to preserve the elastic matrix [30]. Surface functionalizing these NPs with a cationic amphiphile (didodecyldimethylammonium bromide or DMAB), which imparts a positive charge to the NP surface and presents a long-chain hydrocarbon, was found to impart the NPs multifunctional properties, independent of the released DOX, that benefited elastic matrix neoassembly and integrity. We showed the NPs to bind elastic fibers via hydrophobic interactions with their surface hydrocarbon chains, and subsequently upregulate protein expression and activity of the elastin crosslinking enzyme lysyl oxidase (LOX), and attenuate MMP-2 synthesis, and MMP-2 and -9 enzyme activities [30]. We attributed these effects to the positive NP surface charge and hydrocarbon groups presented by the pendant DMAB molecules. While the positive charge on the surface of the NPs has been shown to facilitate their uptake into the arterial wall [31], in this study, we have sought to improve such uptake by rendering the NPs responsive to an applied external magnetic field by incorporating superparamagnetic iron oxide nanoparticles (SPIONs) within. Recent studies by other groups have demonstrated the 
effectiveness of this strategy to deliver drugs to target tissues, particularly deeply situated ones, with reduced systemic side-effects $[32,33]$.

This manuscript represents the first step towards evaluating the utility of these magneticallyresponsive PLGA NPs as vehicles for targeted delivery of DOX to AAAs for effecting regenerative elastic matrix repair. We investigated the effects of SPION incorporation on physical properties of the DOX NPs, their loading and release of DOX, and effects on elastic matrix neoassembly and proteolytic activity in cultures of SMCs isolated from elastase-induced rat AAAs (EaRASMCs). We also carried out preliminary ex vivo studies to demonstrate benefits of magnetic targeting of the NPs to their uptake and retention in the wall of proteolyticallydisrupted vessels in the presence of an external applied magnetic field. The work presented in this manuscript will guide future in vivo studies to assess long-term NP retention and therapeutic effect in a preclinical rat AAA model.

\section{METHODS}

\subsection{Formulation of DOX and DOX-SPION PLGA NPs}

Poly-lactic-co-glycolic acid (PLGA; 50:50 lactide:glycolide; inherent viscosity = 0.95-1.2 $\mathrm{dL} / \mathrm{g}$ in hexafluoroisopropanol; Durect Corporation, Birmingham, AL) was used for the formulation of a) blank NPs (DOX- and SPION-free), b) DOX NPs (loaded with DOX only) and DOX-SPION NPs (loaded with DOX and SPIONs). All NPs were formulated using a double emulsion solvent evaporation technique, using 1\% w/v DMAB (Sigma-Aldrich) as the stabilizer, as described in prior studies by our group [30]. Studies by other groups [32, 34] have also 
utilized this formulation method for the encapsulation of SPIONs, as well as co-encapsulation of a drug along with SPIONs, within PLGA NPs.

Briefly, PLGA was dissolved in chloroform (Fisher Scientific, Fairlawn, NJ) at a concentration of $\sim 2.5-3.0 \% \mathrm{w} / \mathrm{v}$. For DOX NPs, an aqueous solution of doxycycline hydrate (Sigma-Aldrich) at a loading of 2 wt.\% ratio of DOX:PLGA was emulsified into the PLGA solution using a probe sonicator (Q500; QSonica LLC, Newtown, CT) for $1 \mathrm{~min}$ on ice, at an amplitude setting of 20\%. Similarly for DOX-SPION NPs, SPIONs (1.0 mg; fluidMAG amine; chemicell $\mathrm{GmbH}$ ) along with 2 wt.\% of DOX in aqueous solution, was emulsified as described above. The water-in-oil emulsion thus obtained was then further emulsified (1 min on ice, 20\% amplitude) into an aqueous solution of $1 \% \mathrm{w} / \mathrm{v}$ DMAB to form the water-in-oil-in-water emulsion. This secondary emulsion was stirred for $16 \mathrm{~h}$ at room temperature, then desiccated for $1 \mathrm{~h}$ under a vacuum to remove any traces of chloroform. NPs formulated were recovered via ultracentrifugation at 35,000 rpm (Beckman L-80, Beckman Instruments, Palo Alto, CA). The NPs were washed twice with nanopure water to remove any traces of DMAB and unencapsulated

DOX and SPIONs, then lyophilized for $48 \mathrm{~h}$ to obtain a dry powder. Suitable precautions were taken to ensure minimal exposure of DOX to light during the formulation and release process, as tetracyclines are well known to be photosensitive [35].

\subsection{Efficiency of DOX encapsulation within NPs}

The supernatant solutions from the NP formulation steps in section 2.1 were pooled for each individual NP formulation. The amount of DOX was quantified by ultraviolet (UV) spectrophotometry (SpectraMax M2, Molecular Devices, Inc., Sunnyvale, CA), as described in 
our previous papers $[30,36]$. All DOX amounts (in the supernatant solutions) were calculated based on the DOX absorbance peak at $270 \mathrm{~nm}$ [37], from a calibration curve based on serial dilutions of a $1.0 \mathrm{mg} / \mathrm{mL}$ solution of DOX. The total amount of DOX encapsulated within the NPs, and the corresponding overall encapsulation efficiencies were estimated by subtracting the total amount of unencapsulated DOX from the total weighed amounts of DOX added during the NP formulation.

\subsection{Measuring size and surface charge of DOX-SPION NPs}

The mean hydrodynamic diameters (or NP sizes) of the NPs were determined in phosphate buffered saline (PBS; pH 7.4) using a dynamic light scattering technique and mean zeta potentials (or surface charge) of the NPs were determined using a phase analysis light scattering technique. A commercial particle-sizing system (PSS/NICOMP 380/ZLS, Particle Sizing Systems, Santa Barbara, CA) was used for both analyses, as described in our previous studies [30, 36].

\subsection{Determining magnetic velocities of DOX-SPION NPs}

The mobility of the DOX-SPION NPs under an applied magnetic field gradient was quantified by direct measurement of their velocity using a technique called cell tracking velocimetry (CTV) that has been developed by the Zborowski and Chalmers laboratories [38]. The CTV apparatus measures the mobility of individual magnetically-labeled cells or magnetic particles, under the application of a constant magnetic field gradient orthogonal to the direction of the gravitational forces. They have utilized several types of calibration microbeads and 
magnetically-labeled cells in prior studies [38-41], to develop, detail and experimentally verify mathematical relationships to define the magnetophoretic mobility of these cells as the velocity of the cells in an applied magnetic field gradient divided by the magnitude of the magnetic gradient. Details on the calculations for the magnetophoretic mobility and magnetic velocity of magnetic particles and cells have been published in prior studies by the Zborowski group [42].

Lyophilized NPs (prepared as described in Section 2.1) were resuspended in PBS (Sigma-Aldrich) prior to CTV analysis, which was carried out via a protocol modified from studies by the Zborowski laboratory [41, 42]. The DOX-SPION NPs were injected into the flow chamber (1.0 mm I.D, square borosilicate glass channel, Vitrocom, Mountain Lakes, NJ) of the CTV apparatus (in the z-direction), perpendicular to gravity (x-direction), using a $5 \mathrm{~mL}$ syringe connected to PEEK tubing. Following this, the valves on both sides of the flow chamber were closed and sufficient time was given for residual fluid motions to completely dampen. A microscope with $5 \times$ objective (BHMJ; Olympus, Tokyo, Japan) with an attached 12-bit, monochrome, CCD video camera (Retiga Exi, QImaging, Burnaby, BC, Canada) was focused on the NPs within the flow chamber, at a location (Region of Interest, ROI) of constant horizontal magnetic force. This magnetic force was generated by the Mk IV permanent magnet assembly (designed in-house by the Zborowski laboratory) that produces a constant product of magnetization and gradient of $\mathrm{B}$, equal to $1.94 \times 10^{6} \mathrm{~T} \cdot \mathrm{A} / \mathrm{m}^{2}$. This is facilitated by a combination of ceramic Grade 5 permanent magnet blocks and low carbon steel yoke and pole pieces, the latter with faces machined to hyperbolic contours. At the region of interest (ROI), field strength $\mathrm{B}=0.105 \mathrm{~T}$ and field gradient $\mathrm{dB} / \mathrm{dz}=8.01 \times 10^{-3} \mathrm{~T} / \mathrm{mm}$. The motion of the DOX-SPION NPs was captured to a computer hard drive as 50-frame sets, using the Video Savant 4.0 Image Capture software (IO Industries, Inc., London, ON, Canada). The image size was $1200 \times 900$ 
pixels $(1.84 \times 1.38 \mathrm{~mm}$, width $\times$ height $)$, and the brightness of each pixel was binarized to 12-bit grey-scale (with $1 \times 1$ binning). The time interval between captured frames was set to 2.0 seconds. The ImagView Software package (developed/written in-house by the Zborowski laboratory), was used to track the cells between frames using algorithms to establish the most probable path. Macros created in Microsoft Excel enabled us to obtain a linear fit of the location vs. time data, to calculate the velocities of individual NPs. Between 400 and 1000 NPs were processed per sample.

\subsection{Characterizing in vitro release of DOX from DOX NPs and DOX-SPION NPs}

Release studies were carried out over 18 days in phosphate-buffered saline (PBS, pH 7.4; Sigma-Aldrich) at $37^{\circ} \mathrm{C}$, on a shaker at $100 \mathrm{rpm}$. Briefly, DOX NPs and DOX-SPION NPs (0.5 $\mathrm{mg}$ ) were resuspended in $1.0 \mathrm{~mL}$ PBS each in $1.5 \mathrm{~mL}$ polypropylene microcentrifuge tubes $(\mathrm{n}=$ 3 per formulation). At each analysis time-point, the samples were centrifuged $(14,000 \mathrm{rpm}$, 30 min, $4{ }^{\circ} \mathrm{C}$; Beckman Microfuge $16 \AA$, Beckman Coulter, Inc.), the supernatants withdrawn for quantification of DOX, and the volume drawn out was replenished with fresh PBS. The amount(s) of DOX released from the DOX- and DOX-SPION NPs were quantified by UV spectrophotometry, as described in previous studies by our group [30, 36]. The UV absorbance of DOX at $270 \mathrm{~nm}$ was calibrated to its concentration using serial dilutions of a $1.0 \mathrm{mg} / \mathrm{mL}$ DOX solution. The DOX standards were incubated under the same conditions as the NP samples to accurately account for any potential time- and temperature-dependent degradation of DOX [43, 44]. 


\subsection{Morphological characterization of NPs}

Transmission electron microscopy (TEM) was performed to qualitatively compare and contrast the morphology of blank, DOX, and DOX-SPION PLGA NPs. Blank, Doxycycline, and DOX-SPION nanoparticles were resuspended in milliQ water at a concentration of $5 \mathrm{mg} / \mathrm{ml}$ and $5 \mu \mathrm{L}$ of this solution was transferred onto a Formvar carbon-coated copper TEM grid (200 mesh size). The nanoparticle-containing grids were air dried for several hours. TEM images were obtained using a FEI Tecnai Biotwin operated at $68 \mathrm{kV}$ accelerating voltage.

\subsection{Isolation and culture of aneurysmal rat aortic SMCs (EaRASMCs) and culture of human umbilical vein endothelial cells (HUVECs)}

EaRASMCs were isolated from aortae excised from adult male Sprague-Dawley rats $(n=$ 3) at 14-days post-AAA induction via elastase infusion, as described in an earlier study by our group [12]. The primary EaRASMCs thus obtained from these tissue explants were expanded in DMEM-F12 cell culture medium (Invitrogen, Carlsbad, CA) supplemented with $10 \%$ v/v fetal bovine serum (FBS; Invitrogen) and 1\% v/v penicillin-streptomycin (PenStrep; Thermo Fisher, South Logan, UT) for 14 days, and passaged upon reaching confluence. In published studies, we have comprehensively characterized the phenotype and matrix synthesis abilities of these cells, and shown them to maintain an activated/diseased phenotype in culture over several passages [12], All animal procedures were conducted with approval of the Institutional Animal Care and Use Committee (IACUC) at the Cleveland Clinic (ARC \# 2012-0810). The Clinic animal facility is AAALAC-approved and has animal assurance (\#A3145-01; Expires 12/31/17). 
Pooled HUVECs were procured commercially (Lonza, Alpharetta, GA) and cultured in EGM-2 medium with added Clonetics EGM Singlequot ${ }^{\circledR}$ supplements (Lonza). Cells in passage 3-5 were used in experiments.

\subsection{Determining cytotoxicity of DOX-SPION NPs}

A LIVE/DEAD ${ }^{\circledR}$ viability assay (Invitrogen) assessed potential cytotoxic effects of the NPs to EaRASMCs. Briefly, EaRASMCs were seeded at a density of 30,000 cells/well in sterile six-well plates (USA Scientific, Ocala, FL) and allowed to adhere for $48 \mathrm{~h}$ in DMEM-F12 cell culture medium supplemented with $2 \%$ v/v FBS and $1 \%$ v/v PenStrep. DOX and DOX-SPION NPs $(0.2 \mathrm{mg} / \mathrm{mL})$ were incubated with EaRASMCs for $48 \mathrm{~h}$, prior to assessing cell viability. Untreated and blank NP-treated cultures served as controls. Stained cell layers were visualized on an Olympus IX51 fluorescence microscope (Olympus America, Center Valley, PA). Six different regions in each replicate culture were assessed.

\subsection{Assessing impact of DOX-SPION NPs on SMC phenotype}

EaRASMCs were seeded on cover slips within wells of a six well plate at a density of 30,000 cells per cover slip. At 5 days of culture, the cells were cultured for 12 hours with DOXSPION NPs at a concentration of $0.2 \mathrm{mg} / \mathrm{ml}$ in DMEM medium containing $10 \% \mathrm{v} / \mathrm{v}$ FBS, or with medium alone (controls). The cell layers were then fixed in ice-cold methanol for 20 minutes, permeabilized with $0.1 \% \mathrm{v} / \mathrm{v}$ Triton-X and blocked with $5 \% \mathrm{v} / \mathrm{v}$ goat serum. The cells were labeled overnight with rabbit anti-rat antibodies (Abcam) for the early stage SMC marker, $\alpha$ smooth muscle actin (a-SMA), midstage SMC markers caldesmon, and smoothelin and for 
MMP-2 and used at 1: $100 \mathrm{v} / \mathrm{v}$ dilution. Expression of the said proteins was visualized using an Alexa fluor 488-conjugated goat anti-rabbit secondary antibody (Abcam; 1: $500 \mathrm{v} / \mathrm{v})$. The cell layers/cover slips were mounted onto glass slides with Vectashield ${ }^{\circledR}$ mounting medium (Vector labs, Burlingame, CA) containing the nuclear dye, (4',6-Diamidino-2-Phenylindole, Dihydrochloride) (DAPI) and visualized on the Olympus IX51 fluorescence microscope. Cell layers treated with the secondary probe alone were imaged as immunofluorescence (IF) controls to confirm lack fluorescence associated with non-specific attachment of the secondary antibody.

\subsection{Assessing DOX-SPION NP-induction of cellular oxidative stress}

Oxidative stress was quantified using an ROS/Superoxide Detection Assay Kit (Abcam). EaRASMCs and HUVECs were separately seeded in a 96 well plate at a density of 20,000 cells per well and cultured in their respective media until the cells were at $\sim 70-80 \%$ confluent $(\sim 48$ hrs). The medium was aspirated and fresh medium added. The cells were simultaneously treated with DOX-SPION NPs $(0.2 \mathrm{mg} / \mathrm{ml})$ and $100 \mu \mathrm{l}$ of ROS/Superoxide Detection Solution (1:2500 v/v dilution of Oxidative Stress Detection Reagent (Green), 1:2500 v/v dilution of Superoxide Detection Reagent (Orange). Cultures deemed positive controls were treated for 30 minutes with the ROS inducer, pyocyanin $(200 \mu \mathrm{M})$ prepared in dimethyl formamide (DMF) prior to incubation with the detection reagent(s). Cultures assayed as negative controls were treated instead with the ROS inhibitor, N-acetyl-L-cysteine, prepared in deionized water at a concentration of $5 \mathrm{mM}$. Following addition of the detection reagents, the cell layers were incubated for 60 minutes at $37^{\circ} \mathrm{C}$. The plate was them read from the bottom using a fluorescent plate reader for fluorescein $\left(\lambda_{\mathrm{ex}}=488 \mathrm{~nm}, \lambda_{\mathrm{em}}=520 \mathrm{~nm}\right)$ and rhodamine $\left(\lambda_{\mathrm{ex}}=550 \mathrm{~nm}, \lambda_{\mathrm{em}}=\right.$ $610 \mathrm{~nm})$. 


\subsection{Design of cell culture experiments}

All cell culture experiments to evaluate the functional effects of the NPs in vitro within EaRASMC cultures were carried out over 21 days, as we have described previously [30, 45]. Briefly, EaRASMCs (passages 2-5) were seeded at a density of 30,000 cells/well in sterile sixwell plates (USA Scientific, Ocala, FL) and allowed to adhere for $48 \mathrm{~h}$ in DMEM-F12 cell culture medium supplemented with $2 \%$ v/v FBS and 1\% v/v PenStrep, in addition to $100 \mathrm{ng} / \mathrm{mL}$ TNF- $\alpha$ (PeproTech, Inc., Rocky Hill, NJ), to stimulate an activated or aneurysmal phenotype. Subsequently, DOX NPs and DOX-SPION NPs ( $\mathrm{n}=6$ per test case) were added to the cells at a concentration of $0.2 \mathrm{mg} / \mathrm{mL}$ and cultured for 21 days. The well-plates treated with DOX-SPION NPs were exposed (for $40 \mathrm{~min}$ ) to an external magnetic field from a linear Halbach array of diametrically polarized magnet cylinders, designed by the Zborowski laboratory [46]

EaRASMCs cultured with blank NPs $(0.2 \mathrm{mg} / \mathrm{mL}$ NP concentration $)$ served as the activeagent controls, while NP-free EaRASMC cultures served as the untreated controls. The cell culture medium was aspirated out from all wells and replenished with fresh medium every 7 days. The NPs were almost all bound to the cell layer and appropriate care was taken to ensure that the NPs were not removed along with the spent medium. At the end of 21 days of culture, the cell layers were harvested appropriately for biochemical assays.

\subsection{DNA assay for cell proliferation}


As described in prior studies by our group [30, 45], the DNA content within the EaRASMC cell layers was measured via a fluorometric assay using the Hoechst 33258 dye (Invitrogen). The EaRASMC cell layers were harvested at day 21 of culture, and at day 1 (prior to addition of NPs; time-control) in NaCl-Pi buffer, sonicated on ice, and DNA content assayed. The cell count in the cultures was determined from the DNA content, assuming $6 \mathrm{pg}$ of DNA/cell [47].

\subsection{Fastin assay for elastic matrix amounts}

The total elastic matrix content deposited by the EaRASMCs over 21 days of culture was quantified using a Fastin ${ }^{\circledR}$ assay (Accurate Scientific and Chemical, Westbury, NY), as described in recent publications by our group [30,45]. The cell layer was harvested and resuspended in PBS. Briefly, a $500 \mu \mathrm{L}$ aliquot of this cell suspension was mixed with $100 \mu \mathrm{L}$ of hot oxalic acid $(1.5 \mathrm{M})$ and digested for $90 \mathrm{~min}$ at $95^{\circ} \mathrm{C}$. Following this, the samples were centrifuged $(10,000$

g, $10 \mathrm{~min}$ ) and the supernatant containing solubilized matrix elastin, collected. Hot oxalic acid $(0.25 \mathrm{M} ; 400 \mu \mathrm{L})$ was then added to the pellet to be digested over a further $60 \mathrm{~min}$ at $95^{\circ} \mathrm{C}$. The solubilized extracts from both digestion steps were pooled, and the total elastic matrix content was quantified using the Fastin assay. The total amounts of elastic matrix calculated were also normalized to their DNA content (see section 2.11), in order to provide an accurate comparison between cultures subject to different treatments.

\subsection{Desmosine assay}


After 21 days of culture, EaRASMC layers were harvested in PBS and pelleted by centrifugation. The cell pellets were hydrolyzed with $6 \mathrm{~N} \mathrm{HCl}$ for $48 \mathrm{~h}$, evaporated to dryness and reconstituted in $400 \mu \mathrm{L}$ of water. The samples were then filtered through a $0.45 \mu \mathrm{m}$ filter and desmosine levels determined using a competitive ELISA assay [48]. Desmosine amounts were normalized to the total protein content in the respective samples, which was measured via a ninhydrin assay [49].

\subsection{Western blots for MMP-2 and -9 protein synthesis}

Western blotting was used to semi-quantitatively assess the levels of MMP-2 and -9 synthesis by EaRASMCs in the presence of the NPs and NP-released DOX, as described in recent studies by our group [30,45]. At 21 days of culture, the EaRASMC cultures were harvested in RIPA buffer (Thermo Scientific) containing Halt ${ }^{\mathrm{TM}}$ protease inhibitor (Thermo Scientific), and assayed for total protein content using a bicinchonic acid (BCA) assay kit (Thermo Scientific). Maximum volumes of sample protein $(15.6 \mu \mathrm{L})$ were loaded onto each lane of a $10 \%$ sodium dodecyl sulfate-polyacrylamide gel electrophoresis (SDS-PAGE) gel (Invitrogen) under reducing conditions, along with a SeeBlue ${ }^{\circledR}$ pre-stained molecular weight ladder (Invitrogen). The gels were transferred onto nitrocellulose membranes (iBlot ${ }^{\circledR}$ Western Blotting System; Invitrogen), following which they were blocked (Odyssey blocking buffer; LICOR Biosciences, Lincoln, NE) for $1 \mathrm{~h}$ at room temperature. Primary antibody labeling was carried out on the membranes for $16 \mathrm{~h}$ at $4{ }^{\circ} \mathrm{C}$ with a rabbit polyclonal antibody against MMP-2 (1:500 dilution; Abcam, Cambridge, MA) and a rabbit monoclonal antibody against MMP-9 (1:500 dilution; Millipore, Inc.), with a mouse monoclonal antibody against $\beta$-actin (1:1000 
dilution; Sigma-Aldrich) as the loading control. Secondary antibody labeling was carried out for

$1 \mathrm{~h}$ at $24^{\circ} \mathrm{C}$ using IRDye ${ }^{\circledR} 680 \mathrm{LT}$ goat-anti-rabbit $\left(1: 15,000\right.$ dilution) and IRDye ${ }^{\circledR} 800 \mathrm{CW}$ goat anti-mouse (1:20,000 dilution) polyclonal antibodies (LI-COR Biosciences). Protein bands were visualized on the immunolabeled membranes via fluoro-luminescent detection, using a LI-COR Odyssey laser-based scanning system. The intensities of the MMP-2 bands on all membranes were quantified using ImageJ software, expressed in terms of relative density units (RDU) and normalized to that of their respective $\beta$-actin (loading control) bands, to enable comparison of different test cases within the same blot. The $\beta$-actin-normalized band intensities for MMP-2 for all NP-treated cultures were further normalized to that of untreated EaRASMC controls, to determine the fold-change in MMP-2 synthesis, and the statistical significance of the differences between them. The results presented in this manuscript were averaged from three replicate gels.

\subsection{Gel zymography for MMP-2 and -9 activities}

Enzyme activities of MMPs-2 and -9 were analyzed using gel zymography, as described in prior studies by our group [30, 45]. Briefly, volumes of the cell layer (harvested in RIPA buffer containing protease inhibitor) equivalent to $12 \mu \mathrm{g}$ of protein were loaded into each lane of a $10 \%$ zymogram gel (Invitrogen). The gels were run for $2 \mathrm{~h}$ at $125 \mathrm{~V}$. Following this, the gels were washed in a buffer containing $2.5 \% \mathrm{v} / \mathrm{v}$ Triton-X-100 for $30 \mathrm{~min}$ to remove any traces of SDS, and incubated in a substrate/development buffer for $72 \mathrm{~h}$ to activate the MMPs. Finally, the gels were stained $\left(45 \mathrm{~min}, 24^{\circ} \mathrm{C}\right.$ ) with a Coomassie Brilliant Blue solution (Thermo Fisher), and then destained for $90 \mathrm{~min}$, until clear bands were visibly apparent against the dark blue background of the gel. The intensities of the MMP-2 and -9 bands (in RDU) for the NP-treated 
EaRASMC cultures were measured using ImageJ software, and normalized to those obtained for NP-untreated control EaRASMC cultures, to determine the fold-change in their activities. Data was acquired from 3 independent replicate gels.

\subsection{IF-based visualization of elastic matrix in cell layer}

IF staining was performed to visualize elastic matrix within EaRASMC layers following culture with the NPs. EaRASMCs were initially seeded at a density of 30,000 cells/well on 42 $\mathrm{mm} \times 42 \mathrm{~mm}$ cover slips (VWR Scientific; Radnor, PA). Following 21 days of culture, the EaRASMC cell layers were fixed with ice-cold methanol. The cell layers were blocked with 5\% v/v goat serum (PAA Laboratories, Etobicoke, Canada) in PBS, prior to incubation with a rabbit anti-rat polyclonal antibody against elastin (Millipore; 1:100 v/v dilution). Elastic matrix was visualized using Alexa Fluor 488-conjugated IgG secondary antibodies (1:1000 v/v dilution;

Invitrogen). The cell layers were mounted with Vectashield ${ }^{\circledR}$ (Vector Labs) containing the nuclear stain DAPI. A minimum of six regions per cell layer from a total of $n=3$ replicate treatments were imaged to assess representative outcomes. Imaging was performed on an Olympus IX51 fluorescence microscope.

\subsection{Assessing integrity of the luminal endothelium in matrix injured vessels}

In anticipation of assessing the benefits of magnetic targeting of DOX-SPION NPs to vessel wall uptake ex vivo in elastic matrix-disrupted vessels described subsequently, we investigated the impact of elastase injury (our model system) on the luminal endothelium, a 
natural barrier between blood and the underlying vessel wall. Porcine carotid arteries (Lampire Biologicals, Pipersville, PA) were infused with either elastase (20 units/ml, Sigma) or 0.9\% v/v sterile saline (treatment control). The arteries were clamped shut at one end and a catheter was used to infuse the solution $(1 \mathrm{ml})$ before clamping the other end shut. The arteries were incubated for 30 minutes at $37^{\circ} \mathrm{C}$. Following incubation, the arteries were washed with PBS and fixed in $4 \% \mathrm{v} / \mathrm{v}$ paraformaldehyde at $37{ }^{\circ} \mathrm{C}$ for 5 minutes before fixation overnight at $4{ }^{\circ} \mathrm{C}$. The tissues were then postfixed in $1 \% \mathrm{w} / \mathrm{v}$ osmium tetroxide $(1 \mathrm{~h})$, dehydrated in a graded ethanol series (50\%-100\% v/v), embedded in Epon 812 resin, sectioned, placed on copper grids, stained with uranyl acetate and lead citrate, and observed on a Hitachi TEM H7600T (High Technologies, Pleasanton, CA).

\subsection{Nanoparticle adherence to, and translocation through cytokine-activated ECs}

HUVECs were seeded onto glass coverslips at a density of 30,000 and allowed to attach overnight at $37^{\circ} \mathrm{C}$. To mimic cytokine-induced injury to the endothelium within the AAA wall, the a subset of HUVEC cultures were stimulated with TNF- $\alpha(10 \mathrm{ng} / \mathrm{ml})$ for 24 hours before 3 hours of coculture with AF633-loaded PLGA NPs. The cells in the non-activated (control) and cytokine-activated cultures were subsequently rinsed repeatedly to remove any unbound NPs. The cells were stained with Vybrant ${ }^{\circledR}$ DiI (Invitrogen), a membrane dye, then fixed with 4\% v/v paraformaldehyde $\left(20 \mathrm{~min}, 4^{\circ} \mathrm{C}\right)$ and mounted onto slides with VectaShield ${ }^{\circledR}$ containing DAPI. A z-stack series of images of the cell layers was generated using a confocal microscope (Leica Microsystems, Wetzlar, Germany) and 3D reconstruction of the overlays was performed on Volocity Imaging Software to visualize the cells and location of the adhered/uptaken fluorescent NPs. 


\subsection{Impact of endothelial cell activation on trans-endothelial macromolecular transport}

A first experiment investigated in HUVEC cultures, the impact of cytokine activation on endothelial morphology. HUVECs were seeded at 30,000 per well on coverslips placed within wells of a 12 well plate. After attachment overnight, the cells were stimulated with TNF- $\alpha$ (10 $\mathrm{ng} / \mathrm{ml}$ ) for 24 hours, or received only growth medium instead (non-ativated controls). Cell layers were then briefly rinsed in PBS, fixed in $4 \% \mathrm{v} / \mathrm{v}$ paraformaldehyde $\left(20 \mathrm{~min}, 4{ }^{\circ} \mathrm{C}\right)$. The cell layers were blocked with 5\% v/v donkey serum (Sigma Aldrich, St. Louis, Missouri) in PBS, prior to incubation with a goat anti-human monoclonal antibody against tight junction-forming transmemberane protein, e-cadherin (R\&D Systems, Minneapolis, MN; 1:100 v/v dilution). The expressed e-cadherin was visualized using Alexa Fluor 488-conjugated IgG secondary antibodies $\left(1: 1000 \mathrm{v} / \mathrm{v}\right.$ dilution; Invitrogen). The cell layers were mounted with Vectashield ${ }^{\circledR}$ (Vector Labs) containing the nuclear stain DAPI and visualized on the Olympus IX51 fluorescence microscope.

A second experiment sought to generate evidence that trans-endothelial macromolecular transport is increased upon cytokine-activation. HUVECs (30,000 each) were seeded onto 1.0 $\mu \mathrm{m}$ PET membrane cell inserts (Greiner bio-one, Switzerland) that had been previously coated with rat tail collagen type I (BD Biosciences, Bedford, MA; $1 \mathrm{mg} / \mathrm{ml}, 37{ }^{\circ} \mathrm{C}, 1 \mathrm{~h}$ ) and placed within wells of a 12-well plate. The cells were cultured to confluence. One set of HUVECs was then stimulated with TNF- $\alpha(10 \mathrm{ng} / \mathrm{ml})$ for 24 hours, while the second set was not stimulated. FITC-Dextran (company, location; $1 \mathrm{mg} / \mathrm{ml}, 150 \mathrm{kDa} \mathrm{MW}$ ) was added to each of these two inserts, and to a third, collagen-coated insert containing no HUVECs (cell-free controls). All inserts were placed within wells of a 12-well plate and incubated at $37^{\circ} \mathrm{C}$ for 3 hours. Following incubation, the medium contained in the lower chamber was removed and the fluorescence 
associated with migrated FITC-dextran, measured using a fluorescent plate reader $\left(\lambda_{\mathrm{ex}}=490 \mathrm{~nm}\right.$, $\left.\lambda_{\mathrm{em}}=525 \mathrm{~nm}\right)$, and compared against controls.

\subsection{Uptake and retention of SPION-loaded PLGA NPs within the wall of porcine carotids ex vivo}

We evaluated the uptake and retention of SPION-loaded PLGA NPs within the proteolytically-disrupted wall of porcine carotid arteries (length $=\sim 5 \mathrm{~cm}$; Lampire Biological Laboratories; Pipersville, PA) following ex vivo infusion. The endothelia of the carotids were disrupted using a guide wire. The distal end of the artery was clamped with a clip, and a 20 $\mathrm{U} / \mathrm{mL}$ porcine pancreatic elastase (Sigma-Aldrich) solution was infused intraluminally using a Scimed 5F catheter (SCIMED Life Systems, Maplegrove, MN). The proximal end of the arteries were clamped and they were incubated with the elastase for $30 \mathrm{~min}$ at $37{ }^{\circ} \mathrm{C}$ (conditions deemed optimal based on pilot studies varying concentrations of elastase and incubation times). The purpose of such incubation was to achieve proteolytic disruption of wall elastic matrix in addition to endothelial damage. The arteries were subsequently washed thrice with sterile PBS and then infused with a suspension of PLGA NPs $(2.0 \mathrm{mg} / \mathrm{ml})$ containing co-encapsulated SPIONs and the fluorescent dye, Alexa Fluor ${ }^{\circledR} 633$ (0.1 mg, AF633; carboxylic acid, succinimidyl ester; Invitrogen), formulated via the double emulsion solvent evaporation technique described in section 2.1. The NPs were infused using a Scimed 5F catheter. Test arterial segments were exposed to a permanent NdFeB magnet (CMS Magnetics, Garland, TX) for $40 \mathrm{~min}$ [50], while control segments were not exposed to a magnetic field. Following this, all arterial segments were flushed thrice with PBS to remove any non-adherent NPs. Retention of 
uptaken fluorescent NPs was visualized via whole tissue imaging using a Bruker Xtreme system (Bruker, Billerica, MA).

Images obtained from this whole tissue imaging technique were analyzed using the ImageJ software, to quantify the relative fluorescence along the length of the carotid arteries. The background fluorescence (discerned from analyses of matrix-injured but saline infused arteries) was subtracted out. The results were plotted in terms of the mean fluorescence value across the width of the artery as a function of the axial distance from the point of cell infusion. Area under the curve was calculated as a measure of the total uptake of fluorescent NPs into the vessel wall. The ratio of the areas under the curve determined for the text and control vessels was determined as a measure of the fold-increase in NP uptake due to magnetic driving force.

\subsection{Statistical analysis}

All experimental data in this manuscript $(n=6$ replicates per condition, unless stated otherwise) represents the mean \pm standard deviation (SD). Statistical significance of differences between mean values for different samples and conditions was evaluated using Student's $t$-test, with $p \leq 0.05$ considered as statistically significant.

\section{RESULTS}

\subsection{Characterization of NP size, surface charge and magnetic velocity}

Blank (DOX- and SPION-free) NPs exhibited a mean hydrodynamic diameter (or hydrated particle size) of $316.1 \pm 6.3 \mathrm{~nm}$, with a mean $\zeta$-potential (or surface charge) of $49.4 \pm$ 
$7.0 \mathrm{mV}$. Encapsulation of DOX (2\% w/w DOX:PLGA) alone resulted in a modest but statististically significant increase in NP size (see Table 1; $p=0.0012$ vs. no blank NPs) but no significant difference in NP charge. Differently, incorporation of both DOX (2\% w/w DOX:PLGA) and SPIONs resulted in NPs whose size and charge were not different from the blank NPs; the size of these NPs were however statistically lower than the DOX NPs ( $p=0.001)$. TEM (Figure 1) showed that NPs continued to maintain a spherical morphology upon loading with DOX alone or DOX together with SPIONs and individual NPs appear roughly similar in size, in their non-hydrated states. The mean encapsulation efficiency for DOX within the NPs was similar to that we have previously published [30], and was not altered upon co-incorporation of SPIONs. The DOX-SPION NPs alone were found to be mobile in the presence of an applied external magnetic field, and exhibited a magnetic velocity of $1.90 \pm 0.02 \mu \mathrm{m} / \mathrm{s}$, as determined by CTV analysis.

\subsection{In vitro DOX release from NPs}

As seen in Figure 2, DOX release from both DOX and DOX-SPION NPs was characterized by an initial burst phase over the first $24-48 \mathrm{~h}$, followed by a slower exponential release phase, as we have shown earlier with DOX NPs [30]. Beyond the $24 \mathrm{~h}$ time point, DOX release from the DOX-SPION was lower than that observed from DOX NPs, despite both types of NP formulations exhibiting relatively similar encapsulation efficiencies (Table 1).

\subsection{Evaluation of cytotoxicity of DOX- and DOX-SPION NPs in vitro}


A LIVE/DEAD ${ }^{\circledR}$ assay indicated hardly any red (dead) cells (Figure 3) in EaRASMC layers following $48 \mathrm{~h}$ of in vitro culture with the tested dose of blank-, DOX-and DOX-SPION NPs.

\subsection{Impact of DOX-SPION NPs on SMC phenotype}

IF images (Figure 4) showed no significant differences between NP-untreated and DOXSPION NP treated EaRASMCs in cellular expression of the SMC marker proteins, a-SMA, caldesmon, and smoothelin. While the cell densities in these representative images are not the same, likely due to uneven distribution of seeded cells within wells, most cells in the control and treated cultures expressed these SMCs markers. Differently, a visibly smaller fraction of cells in the field of view were seen to express MMP 2 in the DOX-SPION NP-treated cultures relative to the untreated cultures.

\subsection{DOX-SPION NP effects on oxidative stress and superoxide generation in EaRASMC and HUVEC cultures}

As seen in Figure 5, neither DOX-NPs nor the DOX-SPION NPs increased oxidative stress and superoxide production over untreated cell controls, in both EarASMC and HUVEC cultures. These levels were significantly lower than that in positive controls and either comparable or lower than that in the negative control cultures. 


\subsection{Effects of DOX released from NPs on EaRASMC proliferation and elastic matrix deposition}

In all cases, the EaRASMCs proliferated actively following seeding (Figure 6A). At the 21 day time point, EaRASMC counts in blank (DOX-free) NP-treated cultures were slightly higher than that in the NP-free control cultures $(p=0.003)$. Cell counts in the DOX-NP treated cultures were lower than in the blank NP-treated cultures $(p=0.04)$, but not significantly different from both the NP-free control cultures and DOX-SPION NP-treated cultures.

Culture with NPs induced significant increases in amounts of elastic matrix deposited by EaRASMCs on an absolute basis (Figure 6B) $\left(p=1.7 \times 10^{-6}\right.$ for blank NP-treated cultures, $\mathrm{p}=$ $7.0 \times 10^{-6}$ for DOX-NP treated cultures, $\mathrm{p}=1.2 \times 10^{-6}$ for DOX-SPION NP-treated cultures) compared to NP-free control cultures. Elastic matrix deposition was also enhanced in the NPtreated cultures on a per-cell basis (Figure 6C; $\left(p=6.5 \times 10^{-6}\right.$ for blank NP-treated cultures, $\mathrm{p}=$ $7.8 \times 10^{-6}$ for DOX-NP treated cultures, $\mathrm{p}=4.3 \times 10^{-6}$ for DOX-SPION NP-treated cultures), compared to NP-untreated control cultures. On an absolute and per-cell basis, elastic matrix amounts were higher in cultures treated with the DOX-NPs than in those treated with the blank NPs ( $p=0.025$ on an absolute basis, and $\mathrm{p}=0.005$ on a per-cell basis). However, DOX-SPION NP-treated cultures, elastic matrix deposition was not different than in the blank NP-treated cultures.

NP-treated cultures contained significantly higher desmosine crosslink content per $\mu \mathrm{g}$ of protein, relative to NP-free cultures $(p=0.001$ for blank NPs, $\mathrm{p}=0.02$ for DOX NPs, $\mathrm{p}=0.001$

for DOX-SPION NPs) (Figure 6D). There was no significant difference in desmosine content between cell layers cultured with the blank NPs, DOX NPs, and SPION-DOX NPs ( $p>0.05$ ). 


\subsection{Effects of DOX- and DOX-SPION NPs on MMP synthesis and activity in EaRASMC cultures}

Figure 7A shows a representative western blot to compare total (active: 62-66 kDa and zymogen forms: $72 \mathrm{kDa}$ ) MMP-2 synthesis by EaRASMCs cultured in presence of the respective NP groups versus the NP-free control cultures. The $\beta$-actin normalized MMP-2 band intensities for the respective NP-treated culture groups are compared to ratios determined for the NP-free control cultures (assigned value of 1.0; Figure 7B). Blank, DMAB-functionalized NPs significantly inhibited MMP-2 synthesis in EaRASMC cultures vs. untreated controls ( $\mathrm{p}=3.8$ $\times 10^{-6}$ ). MMP-2 synthesis was even lower in cultures treated with the DOX- and DOX-SPION NPs further caused a significant reduction in MMP-2 synthesis $\left(\mathrm{p}=4.3 \times 10^{-11}\right.$ for DOX NPs, $\mathrm{p}=$ $1.8 \times 10^{-8}$ for DOX-SPION NPs versus NP-free controls and $\mathrm{p}=1.3 \times 10^{-5}$ for DOX NPs, $\mathrm{p}=$ 0.001 for DOX-SPION NPs versus blank NP-treated cultures). Compared to the DOX-NPs, the DOX-SPION NPs were slightly less inhibitory towards MMP-2 synthesis $(p=0.01)$ (Figure 2). The bands for MMP-9 were absent or too faint to be quantified reliably [30], in all cases.

Figure 8 shows that enzyme activities of MMPs-2 and -9 in the NP-treated cultures were significantly lower $\left(\mathrm{p}=0.0004\right.$ and 0.0003 for blank NP-treated cultures, $\mathrm{p}=5.4 \times 10^{-5}$ and 0.0001 for DOX NP-treated cultures, $\mathrm{p}=0.0002$ and $6.7 \times 10^{-6}$ for DOX SPION NP-treated cultures, for MMP-2 and -9 , respectively) than that in the NP-free control cultures (band intensity set to unity). The DOX- and DOX-SPION NPs were significantly more inhibitory to both MMP-2 ( $\mathrm{p}=0.04$ for DOX NPs and $\mathrm{p}=0.03$ for DOX-SPION NPs) and MMP-9 $(\mathrm{p}=0.03$ and $\mathrm{p}=0.02$, respectively), compared to the blank NPs. No significant differences in MMP-2 
and MMP-9 enzyme activities were noted between the DOX- and DOX-SPION NP- treated cultures $(p>0.05)$.

\subsection{Immunofluorescence detection of elastin in EaRASMC layers}

Figure 9 shows representative immunofluorescence (IF) images of elastic matrix (green fluorescence) deposited in 21-day cultures of NP-treated and -untreated EaRASMCs. Cultures designated as IF labeling controls (no primary antibody) did not show green fluorescence, indicating the lack of any non-specific binding of the secondary antibody. The NP-treated cultures, especially the DOX NP and DOX-SPION NP-treated cultures exhibited significantly higher green fluorescence than did the NP-untreated cell layers. Elastic matrix deposition in the DOX NP-treated cultures was however visibly higher than in the DOX-SPION NP-treated cultures.

\subsection{Impact of matrix injury and cytokine activation on endothelial layer integrity and trans-endothelial macromolecular transport}

TEM (Figure 10) showed presence of an intact endothelial layer in saline-infused porcine arteries (10A) but significant loss of endothelial cells in elastase-injured arteries (10B). Cytokine activation of confluent HUVEC cultures, mimicking events in the AAA wall, resulted in disruption of the tight junctions characteristic of healthy ECs to create large gaps spanning several microns, between the cells (10C). Confocal imaging showed fluorescent NPs to adhere to and aggregate on the surface of these cytokine-activated HUVECs (10D-1). Image slices of the cell indicated in panel 10D-1, shown at two different planes, one on the surface of the cell (10D- 
2) and another through the cell interior (10D-3) indicate the presence of cells (red) at those locations. The image in panel 10D-4 is a 3-D reconstruction of these serial confocal images, clearly showing the translocation of the NPs (red) through the gap between two cells.

Our transwell diffusion study conducted on FITC-Dextran, showed it to migrate through an intact HUVEC layer, through the pores of the collagen-coated insert, into medium contained in the lower chamber of the transwell system. TNF- $\alpha$ activation of the HUVECs significantly increased FITC-Dextran diffusion into the lower chamber relative to the non-activated condition.

\subsection{Uptake and retention of SPION-loaded NPs ex vivo within proteolytically-disrupted wall of porcine carotid arteries}

Figure 11A shows that in the presence of an applied external magnetic field, uptake and retention of SPION-loaded PLGA NPs (fluorescently labeled with AlexaFluor 633; red) in the

proteolytically-disrupted wall of porcine carotid arteries is significantly greater than in control arteries not exposed to the magnetic field gradient. While the basal fluorescence in control arteries was relatively uniform across their length, that in the test arteries showed greater spatial variability, with some regions showing hyper-saturated fluorescence signals (white).

The plots in Figure 11B show the mean fluorescence measured across the width of the test and control arteries and plotted as a function of the distance from the infusion point. The areas enclosed by the respective curves are indicative of the relative uptake and retention of the SPION-loaded PLGA NPs; arteries exposed to an applied magnetic field gradient showed $1.84 \pm$ 0.34 fold higher NP uptake relative to the control arteries not exposed to a magnetic field. 


\section{DISCUSSION}

In earlier publications, we showed that predictable, and sustained release of DOX release at low micromolar doses from biodegradable PLGA NPs to significantly augment elastic matrix neoassembly by cultured rat aneurysmal SMCs $[30,51]$. This was attributed to surface functionalization of these polymer nanocarriers with a cationic amphiphile (DMAB), which enabled the NPs to bind to forming elastic fibers within the cell layers, via the auspices of the hydrocarbon chains presented by DMAB, and thus release the DOX in close proximity to the cells for greater effect. Independent of the effect of released DOX, we determined that the DMAB-functionalized PLGA nanoparticles themselves were able to stimulate crosslinked elastic matrix deposition. This was attributed to the positive charge imparted to the NP surface by the cationic DMAB moieties, which served to recruit the anionic elastin crosslinking enzyme, lysyl oxidase (LOX) generated by cells to augment crosslinking of elastin precurors into matrix structures [30]. Functionalizing the NP surface with DMAB and its analogs was also shown to impart the nanocarriers significant anti-MMP properties as well, which was attributed to the positive NP charge and long-chain hydrocarbons presented by the pendant DMAB moieties. The anti-MMP effects of DMAB (and its analogs) served to augment the anti-proteolytic effects due the released DOX.

Although the cationic surface modification of the NPs is known to facilitate their uptake and into arterial wall [31], in this work, we sought to improve targeting of the DOX-NPs to the AAA wall by rendering them magnetically responsive in the presence of an applied external magnetic field. The strategy of magnetic targeting of drug-loaded NPs was first developed as a modality to enhance delivery of anti-cancer drugs to target tissues, while reducing their systemic biodistribution [32, 33]. SPIONs, which we incorporate within our polymer NPs in order to 
render them magnetically responsive, have been widely used as contrast agents for magnetic resonance imaging (MRI) of AAAs [52-54]. The purpose behind our incorporation of SPIONs into our DOX-NPs is to enable them to be guided from the lumen of the AAA segment to the AAA wall for uptake, using an external applied magnetic field. In this context, understanding how SPION coincorporation within the DOX-NPs affects NP physical properties and the promatrix regenerative and anti-proteolytic outcomes attributed to the DMAB-functionalized polymer NPs and released DOX, is critical towards gauging their continued therapeutic utility.

As seen in Table 1, the size and surface charge ( $\zeta$-potential) of the PLGA NPs (blank and DOX NPs) were consistent with our published data [30] and were unaffected by co-incorporation of SPIONs. These results suggest that the DOX-SPION NPs continue to be within an appropriate size range $(200-500 \mathrm{~nm})$ that we previously showed to ensure their exclusion by vascular SMCs to the extracellular space [30] where they would serve to influence elastic fiber neoassembly. Supporting this, our TEM images (Figure 1) show blank NPs, DOX-NPs and DOX-SPION NPs, in the non-aggregated state, to exhibit a similar spherical morphology and size, although these do not represent the true (hydrodynamic) diameters of the particles since they are not hydrated. Although the DOX-SPION NPs shown in Figure 1 appear aggregated, such aggregates were not limited to these NPs and were commonly seen with the other NP formulations as well. Overall, these results suggest that SPION coincoproation within DOX NPs minimally alters their physical properties.

Our studies showed even a weak magnetic field (field strength $=0.105 \mathrm{~T}$ and field gradient $=8 \times 10^{-3} \mathrm{~T} / \mathrm{mm}$ ) to impart sufficient magnetic mobility to the DOX-SPION NPs so as to overcome limitations to their migration, imposed by fluid flow [42]. Thus, when this technology is clinically translated, it is expected that the strong magnetic fields $(\sim 3 \mathrm{~T})$ that can 
be generated by clinical MRI scanner systems will be more than sufficient a driving force to efficiently and very rapidly draw intraortally infused DOX-SPION NPs to the AAA wall.

The steady state concentrations of DOX generated from our DOX-NPs (Figure 2) were consistent with that we reported previously, when tested under identical release conditions [30]. These steady state DOX concentrations continued to be below the threshold dose $(10 \mu \mathrm{g} / \mathrm{ml})$ at which we (in unpublished studies) and others $[26,27]$ have shown viability of SMCs to be compromised, and SMC proliferation and elastic matrix neoassembly to be inhibited, both in vitro and in vivo. Although the theoretical DOX loadings and efficiencies of DOX encapsulation (Table 1) within the DOX-NPs and DOX-SPION NPs were near-identical, DOX release from the DOX-SPION NPs was found to be lower. One possibility is that co-encapsulation of SPIONs within the DOX-NPs reduces effective porosity of the polymer matrix to in turn limit indiffusion of aqueous medium and likewise, outward diffusion of solubilized DOX.

Sustained exposure to very high magnetic fields can potentially result in super magnetization of paramagnetic SPIONs to cause their rapid vibration and disruption of the polymer nanoparticles in which they are embedded with adverse implications to drug release. That said, the magnetic fields we have shown to impart magnetic mobility to our SPION-DOX NPs is rather low $(0.1 \mathrm{~T})$ and unlikely to exceed $1 \mathrm{~T}$ even in a clinical setting. We have also estimated that in vivo, a magnet exposure time of less than 3 minutes would be required to drive NPs infused into a flow-occluded aorta to the AAA wall. Accordingly, we do not expect NP integrity to be compromised and hence expect drug release to be maintained as before. We will assess this in our ongoing in vivo study. 
The NPs, both those containing DOX alone or both DOX and SPIONs had no adverse effect on EaRASMC viability (Figure 3) at the tested NP doses, which corresponded to that we showed in our prior published study to augment elastic matrix deposition/crosslinking and to inhibit MMPs even in the absence of DOX loading. Likely, the NPs are not cytotoxic since their surface charge is below the threshold charge $(>55 \mathrm{mV})$ we have determined for cytotoxicity to EaRASMCs (unpublished data). Likely, due to their exclusion in the extracellular space, DOXSPION NPs did not visibly alter phenotype of cocultured EaRASMCs; cellular expression of key SMC phenotypic markers was not visibly different from the NP-untreated cells (Figure 4). However, fewer among the EaRASMCs cultured with the DOX-SPION NPs expressed MMP 2 relative to the NP-untreated EaRASMC group. This outcome is consistent with our published findings as to the anti-proteolytic effects of our DOX-NPs [30]. The low cytotoxicity of the DOX-SPION NPs may also related to the neutral charge on the encapsulated SPIONs. Due to this, they are not expected to induce intracellular lysosomal damage and cellular apoptosis like anionic NPs [55] even upon their eventual release from the PLGA NP as the polymer matrix degrades. Supporting this, we show in Figure 5, that oxidative stress and superoxide levels in EaRASMC and HUVEC cultures did not increase upon exposure to either DOX NPs or DOXSPION NPs. In these cultures, oxidative stress and ROS levels remained significantly below levels measured in positive control cultures and in most cases at or below levels seen in even the negative control cultures. Taken together, these results indicate that our DOX-SPION NPs, at the tested doses do not compromise cell viability or phenotype.

At the stated NP dose, neither the blank NPs nor the DOX- and DOX-SPION NPs had any inhibitory effects on EaRASMC proliferation levels (Figure 6A), which may be attributed to their lack of cytotoxic effects as also near-steady state release of DOX at doses less than earlier 
specified cell proliferation-inhibitory dose range $(5-10 \mu \mathrm{g} / \mathrm{ml})$. Most important, co-encapsulating SPIONs within the DOX NPs had no effect on proliferation of cocultured EaRASMCs.

Our Fastin analysis data shown in Figures 6B and 6C indicate that our NPs have significant pro-elastogenic effects on EaRASMCs, results we qualitatively confirmed by IF staining (Figure 9). Consistent with our previously published findings [30], DMAB-surface functionalized nanocarriers were found to provide a pro-elastogenic stimulus to EaRASMCs independent of the effects of the released DOX. We had previously [30] attributed this effect to the cationic NP surface charge imparted to the NP surface by DMAB, and subsequent recruitment and localized crosslinking activity of cell-generated lysyl oxidase enzyme (LOX; negatively charged at physiological $\mathrm{pH}$ [56]) via opposite charge interactions. This results in more effective crosslinking of cell-secreted tropoelastin (elastin precursor) molecules in the vicinities of the cells into a mature fiber-based matrix. The hydrophobic chains of DMAB can also bind to hydrophobic domains within tropoelastin molecules, to facilitate their nucleation and change their conformation to expose their lysine side chains for more effective crosslinking by LOX [56]. Additionally, the net positive charge of the complex comprising the tropoelastin molecules bound to the DMAB can also preserve newly regenerated elastic matrix by electrostatically repelling cationic elastases expressed in the diseased aneurysmal microenvironment. As we noted previously [30], DOX release from the PLGA NPs further augmented elastic matrix deposition relative to the NP-free cultures. Co-incorporation of SPIONs within the DOX-NPs was found to lessen this augmentary effect such that elastic matrix amounts in DOX-SPION NP-treated cultures was not significantly greater than in the blank NPtreated cultures, although still much higher than in the NP-untreated control cultures. One possible explanation for this is that steady state DOX doses released from the DOX-SPION NPs 
were nearly $25 \%$ lower than that released from the DOX-NPs for similar DOX loadings. This emphasizes the need to augment either the DOX-SPION NP concentration itself, or alternately, DOX loading within our DOX-SPION NPs to be able to generate the equivalent of the proelastogenic, steady state DOX doses released from the DOX-NPs we analyzed in this study.

Formation of desmosine and isodesmosine crosslinks [57] via mediation of LOX, is a reliable measure of a mature elastic matrix [58, 59]. The results from our desmosine assay (Figure 6D) concur with the results of the Fastin assay (Figures 6B and 6C) in indicating that the NPs have pro-elastogenic effects on EaRASMCs. These results are also consistent with our published observations of the stimulus provided by DOX-free, cationic amphiphile-surface modified NPs (both DMAB and its homologs) to crosslinking of elastic matrix in EaRASMC cultures [30]. However, it remains unclear as to why DOX released from the DOX NPs in this study, did not further augment blank NP effects stimulating desmosine crosslinking, despite increasing elastic matrix amounts. Since elastic matrix assembly is a highly complex process [60], of which the crosslinking aspect represents but one step, it is certainly possible that LOXmediated crosslinking is unaffected in the dose range of the released DOX, and that higher DOX loading within the NPs, or alternately a higher concentration of DOX-NPs $(>0.2 \mathrm{mg} / \mathrm{ml})$ may be necessary to improve the elastin crosslinking aspect. Our results in Figure 6D however show that SPION co-incorporation within DOX-NPs does not compromise their overall stimulatory effects on elastic matrix crosslinking in EaRASMC cultures versus NP-free control cultures.

Inhibiting protein synthesis and enzyme activities of elastolytic MMPs -2 and -9 , which are chronically over-expressed within AAAs (and in EaRASMC cultures) [61, 62], is critical to the ensuring long-term benefit of induced elastic matrix regenerative repair. DOX has demonstrated its efficacy in attenuating AAA growth in animal models of AAAs [19, 20, 28, 63], 
as well as in humans [14], via its inhibition of MMP-2 and -9. DOX chelates $\mathrm{Zn}^{2+}$ or $\mathrm{Ca}^{2+}$ ions in the catalytic domain of the active site of MMPs, thereby denaturing/degrading them $[64,65]$ and rendering them inactive. As observed in our prior studies with DOX-loaded PLGA NPs [30], in addition to the effects of DOX, surface functionalization of the NPs with DMAB significantly inhibits MMP-2 synthesis (Figure 7) and MMP-2 and -9 activities (Figure 8). These effects may be attributed to a) the inactivation of negatively charged amino acid residues in the active site of MMPs via electrostatic interactions [66-68] with the cationic chains of DMAB and b) steric blockade of the active site by the long, hydrophobic chains of DMAB [66-68]. Our results in Figures 7 and 8 also show that DOX-SPION NPs resemble the DOX-NPs in inhibiting MMP synthesis and activity versus the NP-free control cultures. The slightly lower inhibition of MMP2 by the SPION-DOX NPs versus the DOX-NPs may be attributed to their lower steady state DOX release doses. Our inability to reliably measure MMP9 protein bands in western blots is consistent with our published observations [12] as to low MMP9 protein synthesis by cultured EaRASMCs in the absence of stimulation by inflammatory cytokines.

In magnetically driving intra-aortally infused DOX-SPION NPs to the AAA wall as we plan to pursue, a key barrier to wall uptake of the NPs could be the ECs lining the AAA wall lumen. When intact and in a healthy state, the ECs are closely placed with characteristic, ecadherin-rich tight junctions between them that prevent molecular transport from blood to the underlying wall connective tissue. That said, there is strong published evidence in both humans [69-71] and animal models of AAAs [72] that the endothelium is highly disrupted in the AAA wall, at least in part due to proteolytic injury and activation by overexpressed cytokines. Our transmission electron micrographs in Figure 10A and B confirm this in showing that in our ex vivo elastase-injured vessel model too, the ECs are mostly disrupted; where retained, large gaps 
were noted between adjacent ECs (not shown). In confluent HUVEC monolayer cultures, we showed that these gaps develop upon cytokine activation of the cells and involve loss of ecadherin-rich tight junctions between cells (Figure 10C). Importantly, these gaps, which span several microns in size, are likely large enough to allow movement of our NPs across. The outcomes of our transwell diffusion experiments with FITC-Dextran (Figure 10E) confirm this. When the confluent HUVEC layer within the insert were cytokine-activated, diffusion of the FITC-Dextran macromolecules across to the medium in the lower chamber was significantly increased. Our confocal images presented in Figure 10D however provide evidence that tranendothelial migration of NPs can also occur by their uptake and passage through the ECs themselves. Likely due to opposite charge interactions, our NPs adhere to the negatively-charged apical surface of ECs and then internalized, likely via pinocytosis to emerge at the basal end. Overall, these experiments provide evidence that the distrupted/activated endothelium is unlikely to be an impediment to migration of DOX-SPION NPs from the AAA lumen into the wall.

Whole tissue imaging (Figure 11) showed low intensity fluorescence from matrix disrupted control arteries infused with DOX-SPION-NPs in the absence of an adjoining weak permanent magnet. This level of basal tissue uptake of the NPs is likely due to functionalization of the NPs with DMAB (a cationic, long chain hydrophobic compound). In fact, DMAB has been shown to facilitate NP uptake and retention into the artery walls [31]. Analysis of the whole tissue images showed $1.84 \pm 0.34$ fold greater uptake and retention of DOX-SPION-NPs into the wall of the arteries in the presence of an adjoining permanent magnet (Figure 11B), relative to their uptake in the absence of the magnet (Figure 11A). However, fluorescence associated with the NPs did not appear uniform across circumference of the artery lumen, likely because the magnet did not uniformly surround the vessel. While the emphasis in this study was not on 
magnet design and magnetic field optimization, we will systematically investigate these aspects also, in the future. The significant levels of retention of DOX-SPION-NPs-associated fluorescence in the test vessels, despite repeat luminal flushing with PBS suggests that the NPs are not superficially adhered to the luminal wall but instead distributed within the wall tissue. Such cell penetration into the wall tissue likely occurs via gaps in the endothelium and the disrupted extracellular matrix, caused by elastase injury. The fluctuation in DOX-SPION-NPassociated fluorescence intensity across the artery length, not including the entry and exit points, may thus be due to uneven/incomplete disruption or/and activation of the lumenal endothelium, and of the underlying ECM resulting from elastase injury. We will investigate these aspects in follow-up in vivo NP delivery studies.

\section{CONCLUSIONS}

Overall, our results demonstrate our abilities to add another dimension of functionality to our DOX-loaded PLGA NPs, in terms of their ability to be magnetically targeted under the application of an external magnetic field, following the co-incorporation of SPIONs. Following SPION encapsulation, the DOX-NPs retained their functional capabilities in terms of enhancing elastic matrix regeneration and inhibiting MMP-2 synthesis and MMP-2 and -9 activities, at levels similar to that observed for DOX NPs. The DOX-SPION NPs were not cytotoxic to EaRASMCs at the doses tested, did not incite cellular oxidative stress and ROS production, and did not phenotypically alter the EaRASMCs, suggesting their safe use. In this work, we have also generated evidence that the luminal endothelium is unlikely to be an impediment to migration of our NPs from the AAA lumen to the AAA wall. Ongoing studies are focused on 
optimizing magnetic field and NP dosing parameters for efficient DOX-SPION NP uptake into the AAA wall in a preclinical small animal model and assessing their retention on site, and/or biodistribution.

\section{ACKNOWLEDGEMENTS}

The authors would like to thank Dr. Judith Drazba and Ms. Cassandra Rogers for assistance with whole tissue imaging and Ms. Sarah Carney, Research Intern for her assistance with the HUVEC culture studies. The authors also acknowledge research funding for this project

from the National Institutes of Health (HL132856), American Heart Association (16 IRG27250113), and National Science Foundation (1508642) awarded to Anand Ramamurthi. 


\section{REFERENCES}

[1] Selle JG, Robicsek F, Daugherty HK, Cook JW. Thoracoabdominal aortic aneurysms: a review and current status. Ann Surg. 1979;189:158-64.

[2] Sakalihasan N, Limet R, Defawe OD. Abdominal aortic aneurysm. The Lancet. 2005;365:1577-89.

[3] Lederle FA, Johnson GR, Wilson SE, Chute EP, Littooy FN, Bandyk D, Krupski WC, Barone GW, Acher CW, Ballard DJ. Prevalence and associations of abdominal aortic aneurysm detected through screening. Ann Intern Med. 1997;126:441-9.

[4] Assar AN. Pharmacological therapy for patients with abdominal aortic aneurysm. Expert Rev Cardiovasc Ther. 2009;7:999-1009.

[5] Rentschler M, Baxter BT. Pharmacological approaches to prevent abdominal aortic aneurysm enlargement and rupture. Ann N Y Acad Sci. 2006;1085:39-46.

[6] Rentschler ME, Baxter BT. Medical therapy approach for treating abdominal aortic aneurysm. Vascular. 2007;15:361-5.

[7] Daugherty A, Cassis LA. Mechanisms of abdominal aortic aneurysm formation. Curr Atheroscler Rep. 2002;4:222-7.

[8] Daugherty A, Cassis LA. Mouse models of abdominal aortic aneurysms. Arterioscler Thromb Vasc Biol. 2004;24:429-34.

[9] Sakalihasan N, Delvenne P, Nusgens BV, Limet R, Lapière CM. Activated forms of MMP2 and MMP9 in abdominal aortic aneurysms. J Vasc Surg. 1996;24:127-33. 
[10] Kielty CM, Sherratt MJ, Shuttleworth CA. Elastic fibres. J Cell Sci. 2002;115:2817-28.

[11] McMahon MP, Faris B, Wolfe BL, Brown KE, Pratt CA, Toselli P, Franzblau C. Aging effects on the elastin composition in the extracellular matrix of cultured rat aortic smooth muscle cells. In Vitro Cell Dev Biol 1985;21:674-80.

[12] Gacchina CE, Deb PP, Barth JL, Ramamurthi A. Elastogenic Inductability of Smooth Muscle Cells from a Rat Model of Late Stage Abdominal Aortic Aneurysms. Tissue Eng. 2011;17:1699-711

[13] Baxter BT, Pearce WH, Waltke EA, Littooy FN, Hallett JW, Kent KC, Upchurch GR Jr, Chaikof EL, Mills JL, Fleckten B, Longo GM, Lee JK, Thompson RW. Prolonged administration of doxycycline in patients with small asymptomatic abdominal aortic aneurysms: Report of a prospective (Phase II) multicenter study. J Vasc Surg. 2002;36:1-12.

[14] Curci JA, Mao DL, Bohner DG, Allen BT, Rubin BG, Reilly JM, Sicard GA, Thompson RW. Preoperative treatment with doxycycline reduces aortic wall expression and activation of matrix metalloproteinases in patients with abdominal aortic aneurysms. J Vasc Surg. 2000;31:325-41.

[15] Ding R, McGuinness CL, Burnand KG, Sullivan E, Smith A. Matrix metalloproteinases in the aneurysm wall of patients treated with low-dose doxycycline. Vascular. 2005;13:290-7.

[16] Mosorin M, Juvonen J, Biancari F, Satta J, Surcel HM, Leinonen M, Saikku P, Juvonen T. Use of doxycycline to decrease the growth rate of abdominal aortic aneurysms: A randomized, double-blind, placebo-controlled pilot study. J Vasc Surg. 2001;34:606-10. 
[17] Prall AK, Longo GM, Mayhan WG, Waltke EA, Fleckten B, Thompson RW, Baxter BT. Doxycycline in patients with abdominal aortic aneurysms and in mice: Comparison of serum levels and effect on aneurysm growth in mice. J Vasc Surg. 2002;35:923-8.

[18] Thompson RW, Baxter BT. MMP inhibition in abdominal aortic aneurysms - Rationale for a prospective randomized clinical trial. In: Greenwald RA, Zucker S, Golub LM, editors. Inhibition of Matrix Metalloproteinases: Therapeutic Applications1999. p. 159-78.

[19] Curci JA, Petrinec D, Liao SX, Golub LM, Thompson RW. Pharmacologic suppression of experimental abdominal aortic aneurysms: A comparison of doxycycline and four chemically modified tetracyclines. J Vasc Surg. 1998;28:1082-93.

[20] Petrinec D, Liao SX, Holmes DR, Reilly JM, Parks WC, Thompson RW. Doxycycline inhibition of aneurysmal degeneration in an elastase-induced rat model of abdominal aortic aneurysm: Preservation of aortic elastin associated with suppressed production of $92 \mathrm{kD}$ gelatinase. J Vasc Surg. 1996;23:336-46.

[21] Hanemaaijer R, Visser H, Koolwijk P, Sorsa T, Salo T, Golub LM, van Hinsbergh VW. Inhibition of MMP synthesis by doxycycline and chemically modified tetracyclines (CMTs) in human endothelial cells. Adv Dent Res. 1998;12:114-8.

[22] Liu J, Xiong WF, Baca-Regen L, Nagase H, Baxter BT. Mechanism of inhibition of matrix metalloproteinase-2 expression by doxycycline in human aortic smooth muscle cells. J Vasc Surg. 2003;38:1376-83.

[23] Uitto VJ, Firth JD, Nip L, Golub LM. Doxycycline and chemically modified tetracyclines inhibit gelatinase A (MMP-2) gene expression in human skin keratinocytes In: Greenwald RA, 
Golub LM, editors. Inhibition of Matrix Metalloproteinases: Therapeutic Potential1994. p. 14051.

[24] Golub LM, Ramamurthy NS, McNamara TF, Greenwald RA, Rifkin BR. Tetracyclines inhibit connective tissue breakdown: new therapeutic implications for an old family of drugs. Crit Rev Oral Biol Med. 1991;2:297-322.

[25] Smith GN, Brandt KD, Hasty KA. Activation of recombinant human neutrophil procollagenase in the presence of doxycycline results in fragmentation of the enzyme and loss of enzyme activity. Arthritis Rheum. 1996;39:235-44.

[26] Bendeck MP, Conte M, Zhang MY, Nili N, Strauss BH, Farwell SM. Doxycycline modulates smooth muscle cell growth, migration, and matrix remodeling after arterial injury. Am J Pathol. 2002;160:1089-95.

[27] Franco C, Ho B, Mulholland D, Hou GP, Islam M, Donaldson K, Bendeck MP. Doxycycline alters vascular smooth muscle cell adhesion, migration, and reorganization of fibrillar collagen matrices. Am J Pathol. 2006;168:1697-709.

[28] Bartoli MA, Parodi FE, Chu J, Pagano MB, Mao DL, Baxter BT, Buckley C, Ennis TL, Thompson RW. Localized administration of doxycycline suppresses aortic dilatation in an experimental mouse model of abdominal aortic aneurysm. Ann Vasc Surg. 2006;20:228-36.

[29] Sho E, Chu J, Sho M, Fernandes B, Judd D, Ganesan P, Kimura H, Dalman RL. Continuous periaortic infusion improves doxycycline efficacy in experimental aortic aneurysms. J Vasc Surg. 2004;39:1312-21. 
[30] Sivaraman B, Ramamurthi A. Multifunctional nanoparticles for doxycycline delivery towards localized elastic matrix stabilization and regenerative repair. Acta Biomater. 2013;9:6511-25.

[31] Labhasetwar V, Song CX, Humphrey W, Shebuski R, Levy RJ. Arterial uptake of biodegradable nanoparticles: Effect of surface modifications. J Pharm Sci. 1998;87:1229-34.

[32] Pouponneau P, Leroux JC, Martel S. Magnetic nanoparticles encapsulated into biodegradable microparticles steered with an upgraded magnetic resonance imaging system for tumor chemoembolization. Biomaterials. 2009;30:6327-32.

[33] Pouponneau P, Leroux JC, Soulez G, Gaboury L, Martel S. Co-encapsulation of magnetic nanoparticles and doxorubicin into biodegradable microcarriers for deep tissue targeting by vascular MRI navigation. Biomaterials. 2011;32:3481-6.

[34] Wassel RA, Grady B, Kopke RD, Dormer KJ. Dispersion of super paramagnetic iron oxide nanoparticles in poly(d,1-lactide-co-glycolide) microparticles. Colloids Surf A Physicochem Eng Asp. 2007;292:125-30.

[35] Honnorat-Benabbou VC, Lebugle AA, Sallek B, Duffaut-Lagarrigue D. Stability study of tetracyclines with respect to their use in slow release systems. J Mater Sci Mater Med. 2001;12:107-10.

[36] Venkataraman L, Sivaraman B, Vaidya P, Ramamurthi A. Nanoparticulate Delivery of Agents for Induced Elastogenesis in 3-Dimensional Collagenous Matrices. J Tissue Eng Regen Med. 2014;DOI: 10.1002/term.1889. 
[37] Mitic SS, Miletic GZ, Kostic DA, Naskovic-Dokic DC, Arsic BB, Rasic ID. A rapid and reliable determination of doxycycline hyclate by HPLC with UV detection in pharmaceutical samples. J Serb Chem Soc. 2008;73:665-71.

[38] McCloskey KE, Chalmers JJ, Zborowski M. Magnetic Cell Separation: Characterization of Magnetophoretic Mobility. Anal Chem. 2003;75:6868-74.

[39] McCloskey KE, Chalmers JJ, Zborowski M. Magnetophoretic mobilities correlate to antibody binding capacities. Cytometry. 2000;40:307-15.

[40] McCloskey KE, Zborowski M, Chalmers JJ. Measurement of CD2 expression levels of IFN$\alpha$-treated fibrosarcomas using cell tracking velocimetry. Cytometry. 2001;44:137-47.

[41] McCloskey KE, Comella K, Chalmers JJ, Margel S, Zborowski M. Mobility measurements of immunomagnetically labeled cells allow quantitation of secondary antibody binding amplification. Biotechnol Bioeng. 2001;75:642-55.

[42] Jing Y, Mal N, Williams PS, Mayorga M, Penn MS, Chalmers JJ, Zborowski M. Quantitative intracellular magnetic nanoparticle uptake measured by live cell magnetophoresis. FASEB J. 2008;22:4239-47.

[43] Haerdi-Landerer MC, Suter MM, Steiner A, Wittenbrink MM, Pickl A, Gander BA. In vitro cell compatibility and antibacterial activity of microencapsulated doxycycline designed for improved localized therapy of septic arthritis. J Antimicrob Chemother. 2008;61:332-40. 
[44] Sunaric SM, Mitic SS, Miletic GZ, Pavlovic AN, Naskovic-Djokic D. Determination of doxycycline in pharmaceuticals based on its degradation by $\mathrm{Cu}(\mathrm{II}) / \mathrm{H}(2) \mathrm{O}(2)$ reagent in aqueous solution. J Anal Chem. 2009;64:231-7.

[45] Sylvester A, Sivaraman B, Deb P, Ramamurthi A. Nanoparticles for localized delivery of hyaluronan oligomers towards regenerative repair of elastic matrix. Acta Biomater. 2013;9:9292302.

[46] Hoyos M, Moore L, Williams PS, Zborowski M. The use of a linear Halbach array combined with a step-SPLITT channel for continuous sorting of magnetic species. J Magn Magn Mater. 2011;323:1384-8.

[47] Labarca C, Paigen K. A simple, rapid, and sensitive DNA assay procedure. Anal Biochem. 1980;102:344-52.

[48] Igoucheva O, Alexeev V, Halabi CM, Adams SM, Stoilov I, Sasaki T, Arita M, Donahue A, Mecham RP, Birk DE, Chu ML. Fibulin-4 E57K knock-in mice recapitulate cutaneous, vascular and skeletal defects of recessive cutis laxa $1 \mathrm{~b}$ with both elastic fiber and collagen fibril abnormalities. J Biol Chem. 2015;290:21443-59.

[49] Starcher B. A ninhydrin-based assay to quantitate the total protein content of tissue samples. Anal Biochem. 2001;292:125-9.

[50] Riegler J, Liew A, Hynes SO, Ortega D, O'Brien T, Day RM, Richards T, Sharif F, Pankhurst QA, Lythgoe MF. Superparamagnetic iron oxide nanoparticle targeting of MSCs in vascular injury. Biomaterials. 2013;34:1987-94. 
[51] Venkataraman L, Sivaraman B, Vaidya P, Ramamurthi A. Nanoparticulate delivery of agents for induced elastogenesis in three-dimensional collagenous matrices. J Tissue Eng Regen Med. 2014.

[52] Neuwelt A, Sidhu N, Hu CA, Mlady G, Eberhardt SC, Sillerud LO. Iron-based superparamagnetic nanoparticle contrast agents for MRI of infection and inflammation. AJR Am J Roentgenol. 2015;204:W302-13.

[53] Sharifi S, Seyednejad H, Laurent S, Atyabi F, Saei AA, Mahmoudi M. Superparamagnetic iron oxide nanoparticles for in vivo molecular and cellular imaging. Contrast Media Mol Imaging. 2015;10:329-55.

[54] Truijers M, Futterer JJ, Takahashi S, Heesakkers RA, Blankensteijn JD, Barentsz JO. In vivo imaging of the aneurysm wall with MRI and a macrophage-specific contrast agent. AJR Am J Roentgenol. 2009;193:W437-41.

[55] Pisanic TR, 2nd, Blackwell JD, Shubayev VI, Finones RR, Jin S. Nanotoxicity of iron oxide nanoparticle internalization in growing neurons. Biomaterials. 2007;28:2572-81.

[56] Kagan HM, Sullivan KA, Olsson TA, Cronlund AL. Purification and properties of four species of lysyl oxidase from bovine aorta. Biochem J. 1979;177:203-14.

[57] Kagan HM, Li WD. Lysyl oxidase: Properties, specificity, and biological roles inside and outside of the cell. J Cell Biochem. 2003;88:660-72.

[58] Gunja-Smith Z. An enzyme-linked immunosorbent assay to quantitate the elastin crosslink desmosine in tissue and urine samples. Anal Biochem. 1985;147:258-64. 
[59] Osakabe T, Seyama Y, Yamashita S. Comparison of ELISA and HPLC for the determination of desmosine or isodesmosine in aortic tissue elastin. J Clin Lab Anal. 1995;9:293-6.

[60] Sivaraman B, Bashur C, Ramamurthi A. Advances in biomimetic regeneration of elastic matrix structures. Drug Deliv Transl Res. 2012;2:323-50.

[61] Davis V, Persidskaia R, Baca-Regen L, Itoh Y, Nagase H, Persidsky Y, Ghorpade A, Baxter BT. Matrix metalloproteinase-2 production and its binding to the matrix are increased in abdominal aortic aneurysms. Arterioscler Thromb Vasc Biol. 1998;18:1625-33.

[62] Longo GM, Xiong WF, Greiner TC, Zhao Y, Fiotti N, Baxter BT. Matrix metalloproteinases 2 and 9 work in concert to produce aortic aneurysms. J Clin Invest. 2002;110:625-32.

[63] Petrinec D, Holmes DR, Liao SX, Golub LM, Thompson RW. Suppression of experimental aneurysmal degeneration with chemically modified tetracycline derivatives. Ann N Y Acad Sci. 1996;800:263-5.

[64] Garcia RA, Pantazatos DP, Gessner CR, Go KV, Woods VL, Villarreal FJ. Molecular interactions between matrilysin and the matrix metalloproteinase inhibitor doxycycline investigated by deuterium exchange mass spectrometry. Mol Pharmacol. 2005;67:1128-36.

[65] Seftor RE, Seftor EA, De Larco JE, Kleiner DE, Leferson J, Stetler-Stevenson WG, McNamara TF, Golub LM, Hendrix MJ. Chemically modified tetracyclines inhibit human melanoma cell invasion and metastasis. Clin Exp Metastasis. 1998;16:217-25. 
[66] Mendis E, Kim M-M, Rajapakse N, Kim S-K. The inhibitory mechanism of a novel cationic glucosamine derivative against MMP-2 and MMP-9 expressions. Bioorg Med Chem Lett. 2009; 19:2755-9.

[67] Tezvergil-Mutluay A, Agee KA, Uchiyama T, Imazato S, Mutluay MM, Cadenaro M, Breschi L, Nishitani Y, Tay FR, Pashley DH. The Inhibitory Effects of Quaternary Ammonium Methacrylates on Soluble and Matrix-bound MMPs. J Dent Res. 2011;90:535-40.

[68] Ganguly B, Banerjee J, Elegbede AI, Klocke DJ, Mallik S, Srivastava DK. Intrinsic selectivity in binding of matrix metalloproteinase-7 to differently charged lipid membranes. FEBS Lett. 2007;581:5723-6.

[69] Franck G, Dai J, Fifre A, Ngo S, Justine C, Michineau S, Allaire E, Gervais M. Reestablishment of the endothelial lining by endothelial cell therapy stabilizes experimental abdominal aortic aneurysms. Circulation. 2013; 127(18):1877-87.

[70] Cafueri G, Parodi F, Pistorio A, Bertolotto M, Ventura F, Gambini C, Bianco P, Dallegri F, Pistoia V, Pezzolo A, Palombo D. Endothelial and smooth muscle cells from abdominal aortic aneurysm have increased oxidative stress and telomere attrition. PLoS One. 2012; 7(4):e35312.

[71] Siasos G, Mourouzis K, Oikonomou E, Tsalamandris S, Tsigkou V, Vlasis K, Vavuranakis M, Zografos T, Dimitropoulos S, Papaioannou TG, Kalampogias A, Stefanadis C, Papavassiliou AG, Tousoulis D. The Role of Endothelial Dysfunction in Aortic Aneurysms. Curr Pharm Des. 2015; 21(28):4016-34. 
[72] Origuchi N, Shigematsu H, Izumiyama N, Nakamura K, Toku A, Muto T. Aneurysm induced by periarterial application of elastase heals spontaneously. Int Angiol. 1998; 17(2):1139. 


\section{FIGURE LEGENDS}

Figure 1. SPION co-encapsulation does not affect morphology of DOX NPs. Transmission electron micrographs of blank PLGA NPs, and DOX-NPs, and DOX-SPION NPs containing 2\% wt/wt ratio of DOX to PLGA. The images show the NPs in all cases to be morphologically similar in being spherical. Since these NPs are not hydrated, no information may be gathered regarding their hydrodynamic sizes, though the NPs appear roughly in the same size range. To be noted, NP aggregates seen in the image of the DOX-SPION NPs, were present in other NP groups as well. Scale bar: $200 \mathrm{~nm}$.

Figure 2. In vitro DOX release profiles generated with DOX and DOX-SPION loaded PLGA NPs. DOX was loaded at a $2 \% \mathrm{wt}:$ wt ratio to PLGA. $(n=3$; mean \pm SD).

Figure 3. Representative images showing LIVE/DEAD® assay stained EaRASMCs following 48 hours of co-culture with DOX-unloaded (blank) and 2\% w/w DOX- and DOX-SPION loaded PLGA NPs (0.2 mg of NPs/ml). Calcein AM-stained live cells fluoresce green. NP-untreated EaRASMCs are shown as controls. Scale bar: $100 \mu \mathrm{m}$.

Figure 4. Representative immunofluorescence images showing lack of adverse effects of DOXSPION NP exposure to expression of SMC phenotypic marker proteins in EaRASMC cultures. However, MMP2 expression by the EaRASMCs was reduced upon coculture with the DOX- 
SPION NPs. Proteins of interest fluoresce green and DAPI-stained cell nuclei appear blue. Scale bars: $100 \mu \mathrm{m}$.

Figure 5. Total oxidative stress and superoxide production measurements in cultures of HUVECs and EaRASMCs upon standalone culture (no NP control), and coculture with 0.3 $\mathrm{mg} / \mathrm{ml}$ of DOX NPs and DOX-SPION NPs loaded with DOX at a $2 \% \mathrm{wt} / \mathrm{wt}$ ratio to PLGA. Also shown are measurements in positive control and negative control cultures. Coculture with the DOX-NPs or DOX-SPION NPs did not increase oxidative stress or superoxide production relative to NP untreated controls and values were similar to or lower than in that in the negative control cultures. * denotes significance of difference versus positive control, deemed for $\mathrm{p}<$ 0.05. \# denotes significance of difference versus negative control, deemed for $\mathrm{p}<0.05$.

Figure 6. Comparative effects of DOX-NPs and DOX-SPION NPs on cell proliferation and elastic matrix deposition and crosslinking in EaRASMC cultures. (A) Proliferation of EaRASMCs in response to DOX released from PLGA NPs with and without SPIONs (0.2 mg of NPs/mL; $2 \%$ w/w of DOX to PLGA). EaRASMCs treated with blank NPs (0\% DOX) were the active agent control. The cell number was calculated based on an estimate of $6 \mathrm{pg}$ of DNA per cell, via a DNA assay, at 1 and 21 days post-seeding. (B) Effects of DOX released from PLGA NPs on total matrix elastin deposition by EaRASMCs on an absolute basis, over 21 days of culture with blank NPs, DOX-NPs and DOX-SPION NPs. NP-untreated cultures served as the treatment controls. (C) Total matrix elastin was normalized to DNA content per cell, to enable a more direct comparison between test cases, by accounting for EaRASMC proliferation. (D) 
Matrix desmosine content, a net measure of the crosslinking of elastic matrix, measured 21 days of culture with blank, DOX- and DOX-SPION NPs. NP-untreated cultures served as the treatment controls. The total desmosine content was normalized to the amount of protein present in the cell layer, so as to enable a more accurate comparison between the different test cases. In all plots, values shown indicate mean $\pm S D$ of measurements made in $n=6$ per case, $*$ denotes $p$ $<0.05$ compared to treatment control; \# denotes $\mathrm{p}<0.05$ compared to blank NP controls.

Figure 7. Effects of DOX- and DOX-SPION-NPs on MMP-2 synthesis in EaRASMC cultures, as analyzed with western blots. (A) Representative image of western blot, with $\beta$-actin bands as loading controls. (B) Fold-differences in beta-actin normalized intensities of the active MMP-2 bands in each treatment test case versus NP-untreated cultures (treatment controls; set to unity; dotted line). Data shown represent mean \pm SD of values obtained by analysis of $n=3$ cultures/case. * and \# denote significance of differences vs. NP-untreated cultures and blank NPtreated cultures (active agent controls), respectively, deemed for $\mathrm{p}<0.05$.

Figure 8. Effect of DOX- and DOX-SPION NPs on MMP-2 and -9 enzyme activities in EaRASMC cultures, as analyzed by gel zymography. (A) Representative image of gel zymogram. (B) Fold-change in band intensities of the active MMP-2 and active MMP-9 band for each treatment test case relative to NP-untreated cultures (treatment controls; assigned value of unity; dotted line). Data shown represent mean \pm SD of values obtained by assay of $n=3$ cultures/case. ${ }^{*}$ and \# denote significance of differences versus NP-untreated cultures and blank NP treated cultures (active agent controls), respectively, deemed for $\mathrm{p}<0.05$. 
Figure 9. Immunofluorescence microscopy images for detection of elastic matrix (green fluorescence) within EaRASMC layers following 21 days of culture in the presence of DOX-and DOX-SPION loaded PLGA NPs. Also shown for comparison are EaRASMC cultures treated with blank NPs (active-agent control), cultured with no NPs (treatment control). Immunolabeling controls received no primary antibodies and exhibited no green fluorescence when treated with the Alexa633-conjugated secondary antibody. DAPI-stained nuclei appear blue. Scale bar: 100 $\mu \mathrm{m}$.

Figure 10. TEM images show presence of an intact lumenal endothelium within healthy porcine carotids, with tight junctions between adjacent cells (A). Elastase-induced injury caused severe disruption of the endothelium, with significant loosening of the underlying sub-endothelial matrix (B). (C) TNF- $\alpha$-activation of a confluent layer of HUVECs similarly resulted in loss of e-cadherin-rich tight junctions between cells (green) to form large gaps several microns in size, through which NP migration is possible. DAPI-labeled nuclei appear blue. Scale bar: $100 \mu \mathrm{m}$. (D) TNF- $\alpha$-activated HUVECs cultured with AF633-loaded NPs (pseudo-colored green) show aggregation on the cell surface (D1). Imaging at different planes through the cell layer (D2,3) showed an increase in NP fluorescence as the plane moved from the apical surface into the cell interior. In addition to NP migration through the cell, the SMPs were shown to move through the gaps between cells (D4). In these images, DiI-labeled cells appear red, and DAPI-stained nuclei, blue. (E) A transwell assay showed diffusion of FITC-Dextran through a layer of HUVECs seeded on collagen coated, porous membrane inserts into medium in a lower chamber to be significantly higher when the HUVECs were stimulated with TNF- $\alpha(\mathrm{E})$. 
Figure 11. SPION loading of PLGA NPs in conjunction with an applied magnetic field significantly increases NP uptake and retention matrix disrupted porcine carotids upon ex vivo infusion. (A) Representative images show distribution of Alexa 633-labeled SPION-PLGA NPs (without and magnet) within porcine carotid arteries as viewed using a Bruker whole tissue imaging system. Curves in panels B and C show mean fluorescence intensities in carotids infused with SPION-PLGA NPs in the presence and absence respectively, of an applied magnetic field. The images were corrected for low background fluorescence seen in non-NP infused carotids prior to quantification of fluorescence intensities. 
Table 1. Properties of NP formulations. * and + indicate significance relative to blank NPs and DOX-SPION NPs respectively, deemed for $\mathrm{p}<0.05$.

\begin{tabular}{|c|c|c|c|c|}
\hline Sample & Size [nm] & $\begin{array}{c}\zeta \text {-potential } \\
{[\mathbf{m V}]}\end{array}$ & $\begin{array}{c}\text { DOX encapsulation } \\
\text { efficiency [\%] }\end{array}$ & Magnetic velocity [ $\boldsymbol{\mu m} / \mathbf{s}]$ \\
\hline Blank NPs & $313.5 \pm 13.0$ & $45.8 \pm 11.6$ & N/A & 0 \\
\hline DOX NPs & $374.9 \pm 14.0^{*,+}$ & $47.5 \pm 12.0$ & $41.6 \pm 1.4$ & $1.90 \pm 0.02$ \\
\hline DOX-SPION NPs & $319.5 \pm 16.4$ & $46.9 \pm 7.8$ & $43.4 \pm 1.1$ & 0 \\
\hline
\end{tabular}




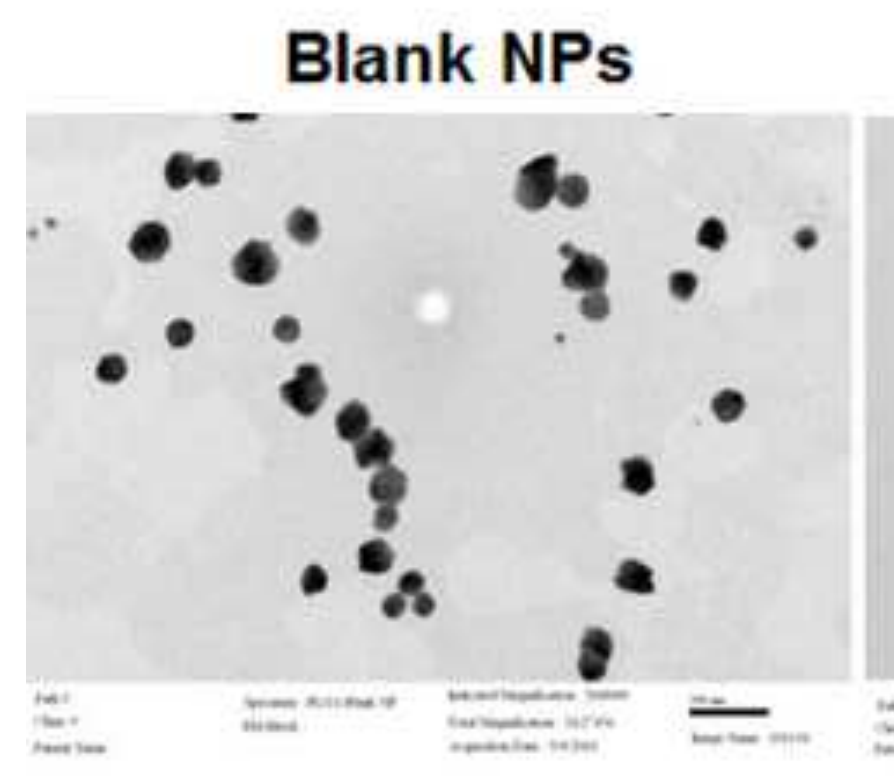

\section{DOX NPs}

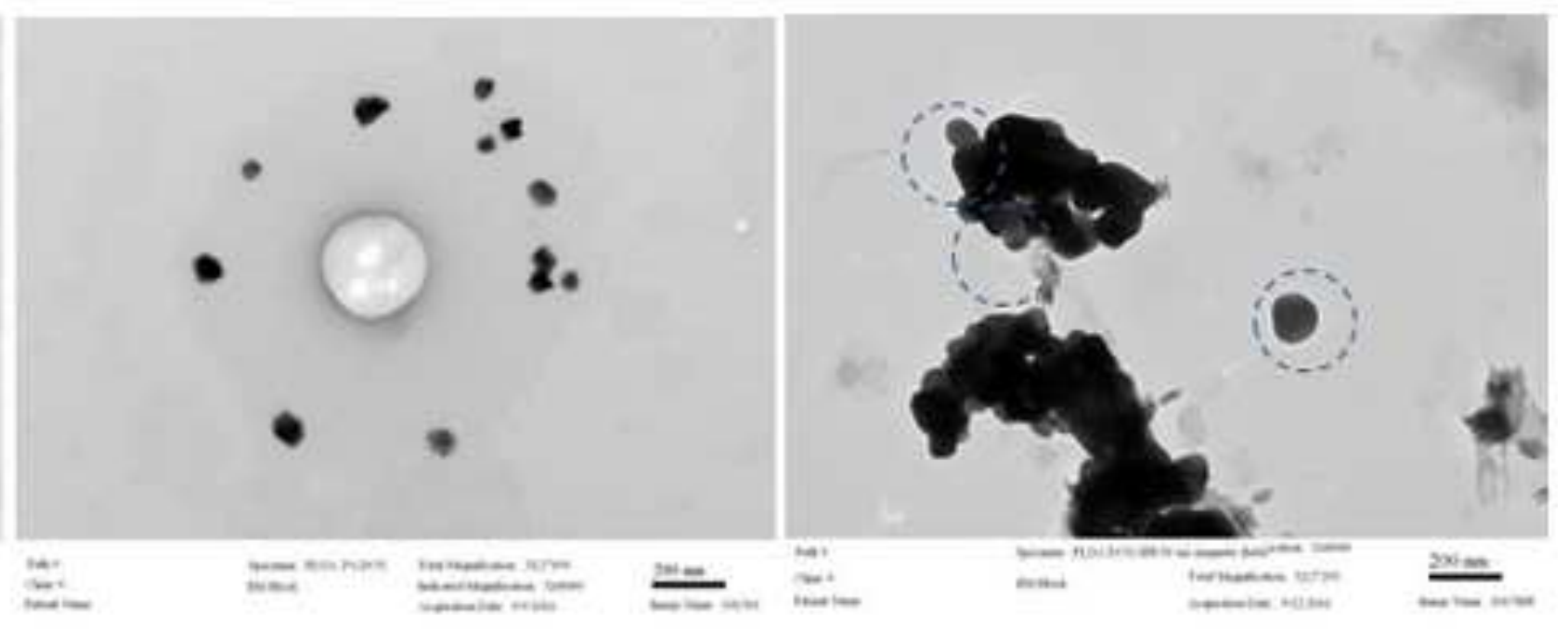

Figure 1 


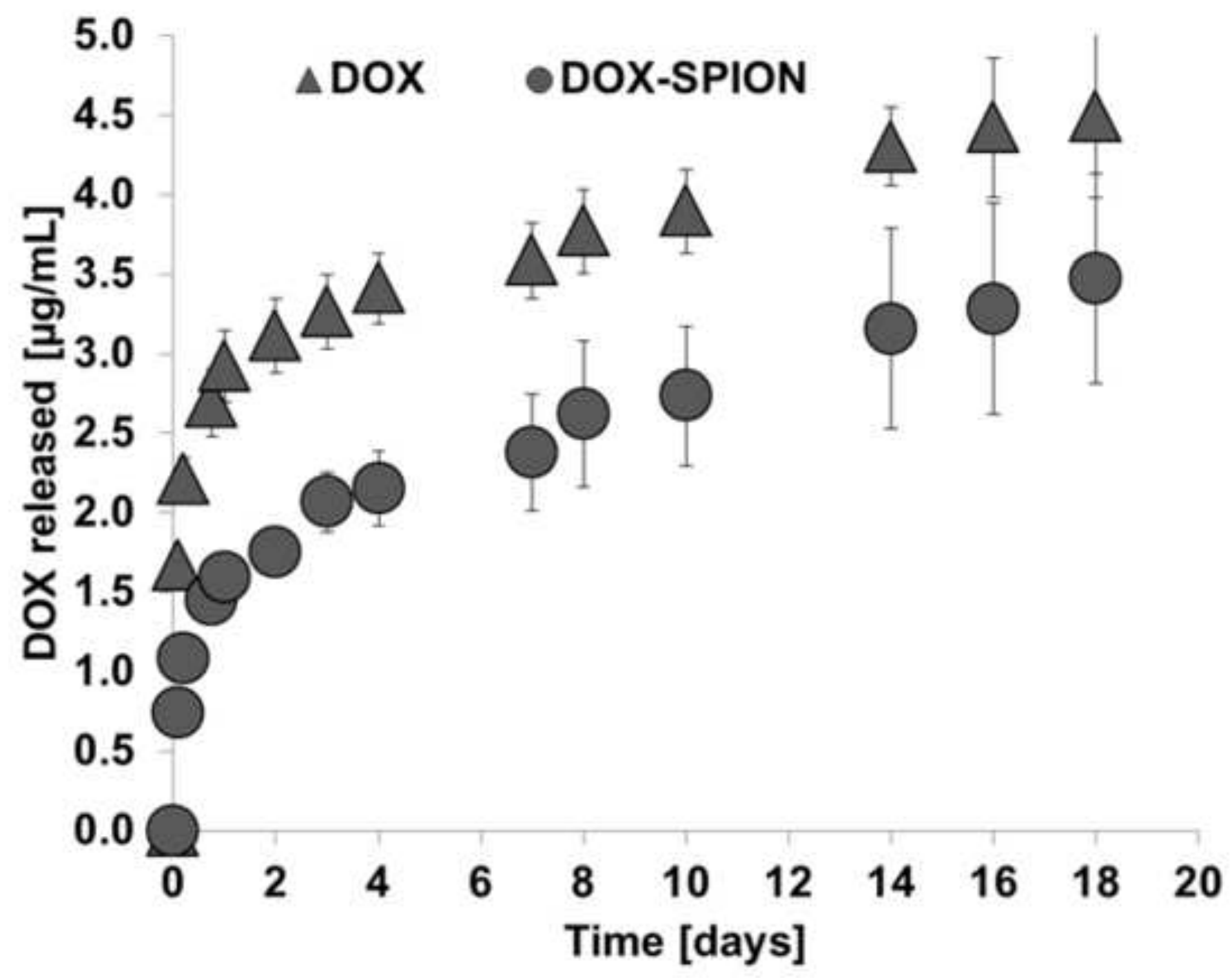

Figure 2 
No NPs

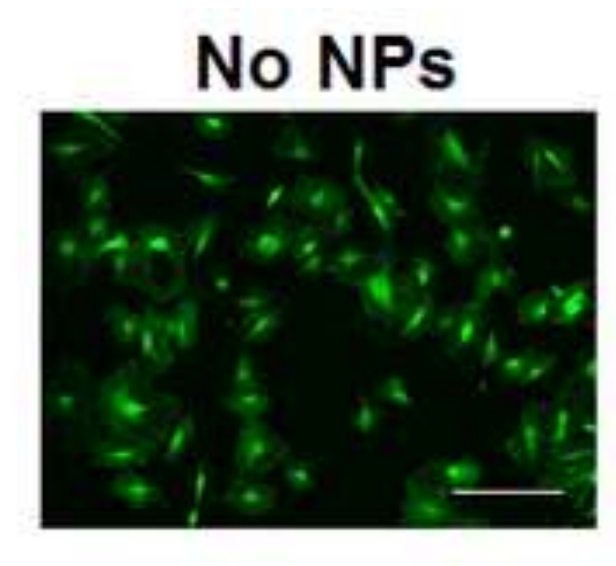

Blank NPs

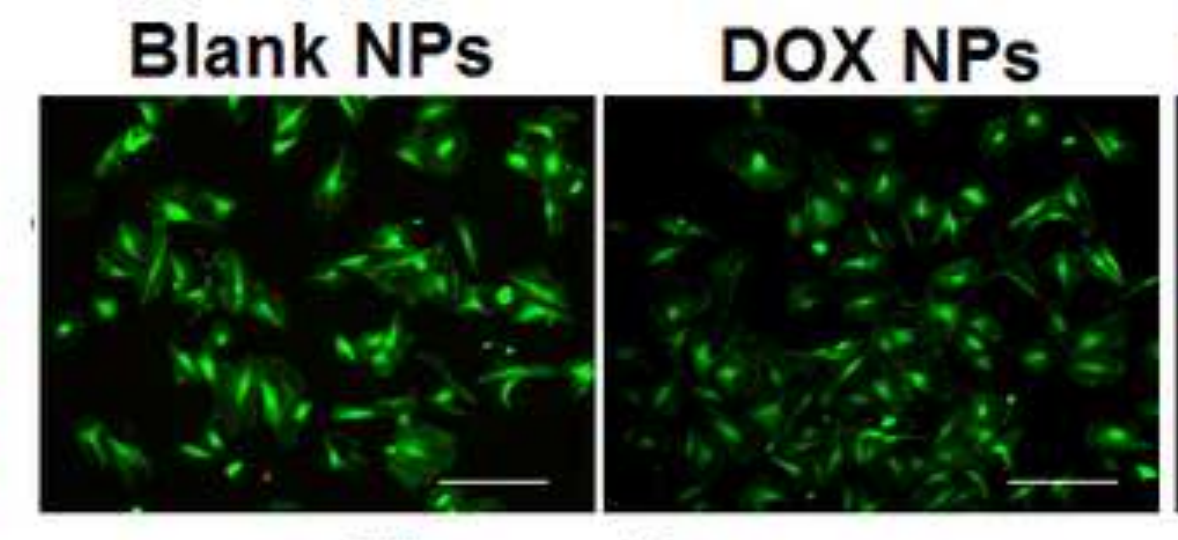

\section{Figure 3}

DOX-SPION NPS

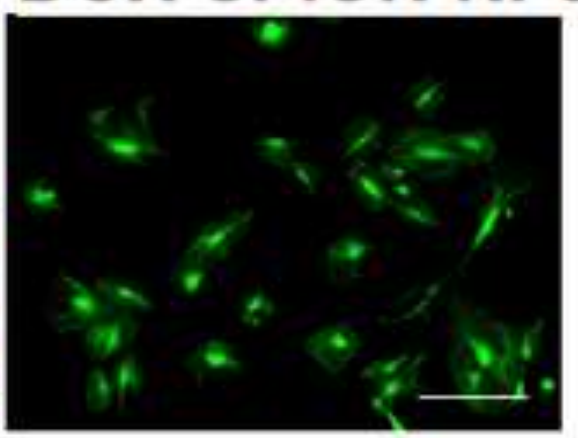

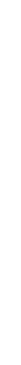

.



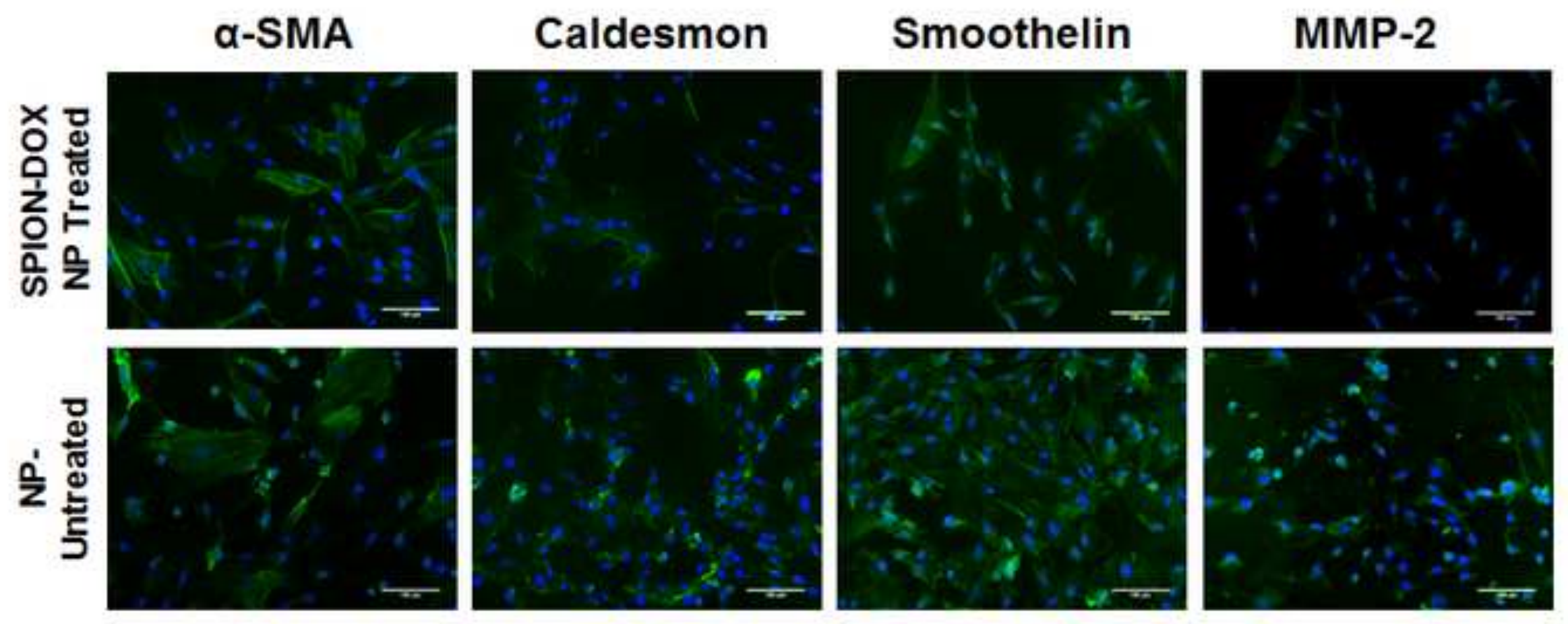

Figure 4 

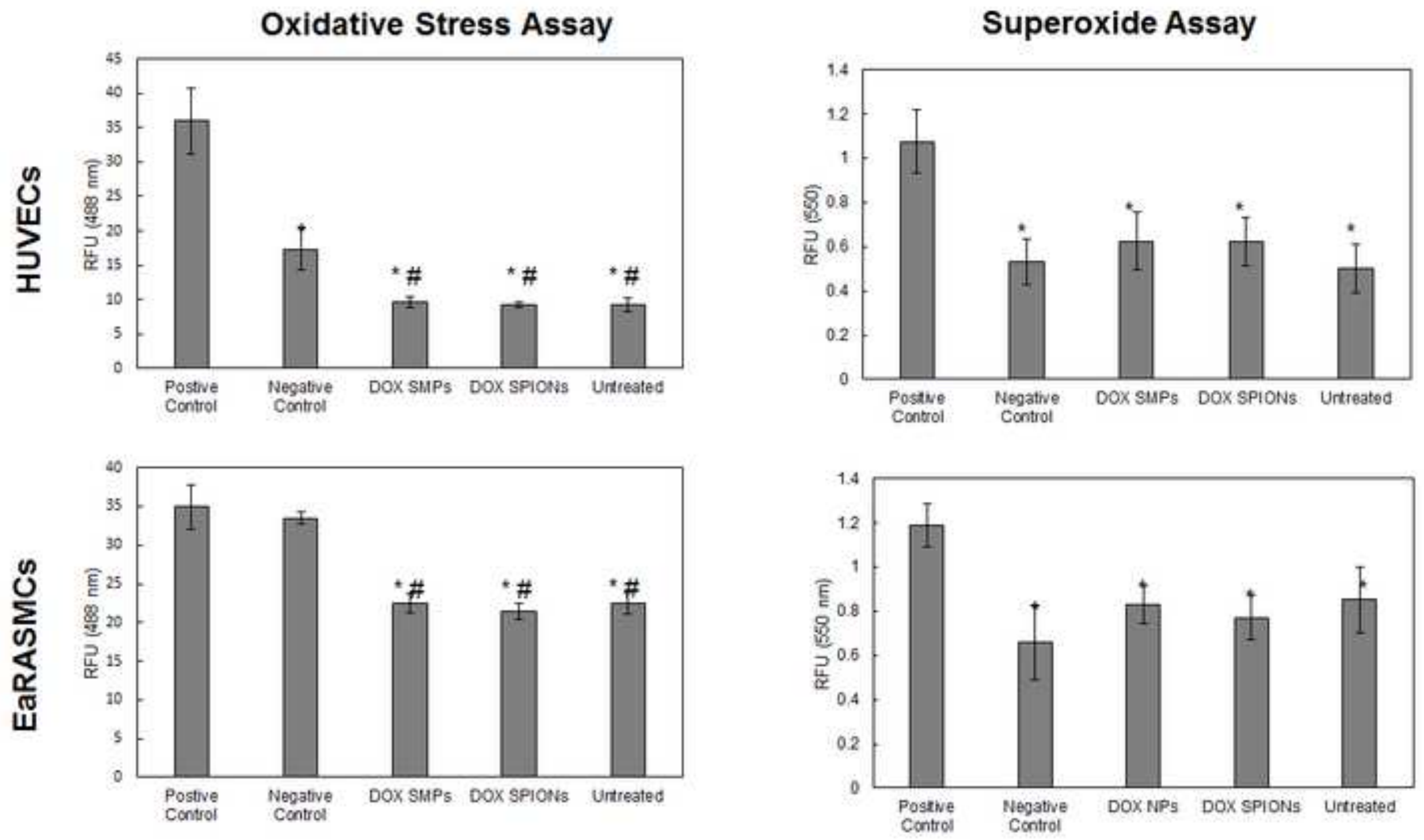

Figure 5 

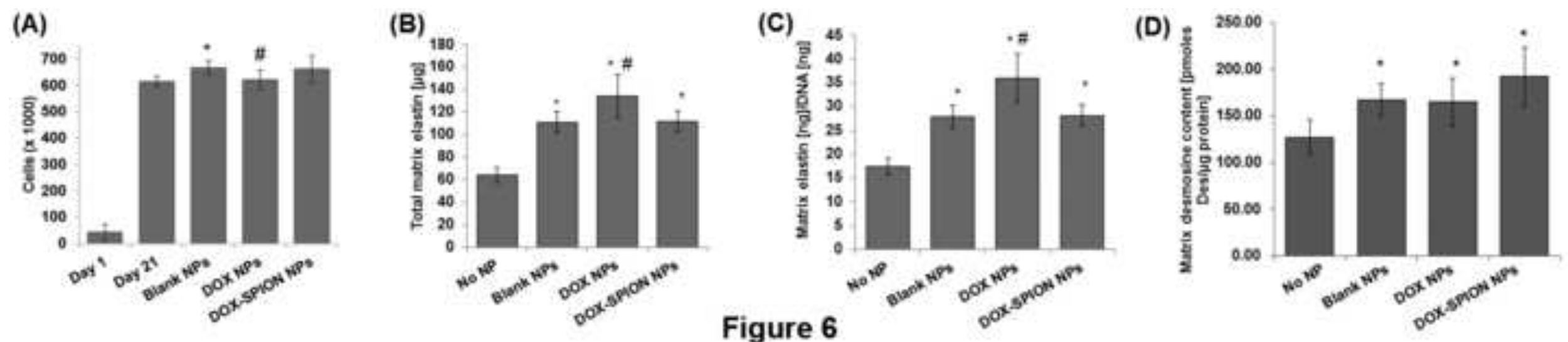

Figure 6 
(A)

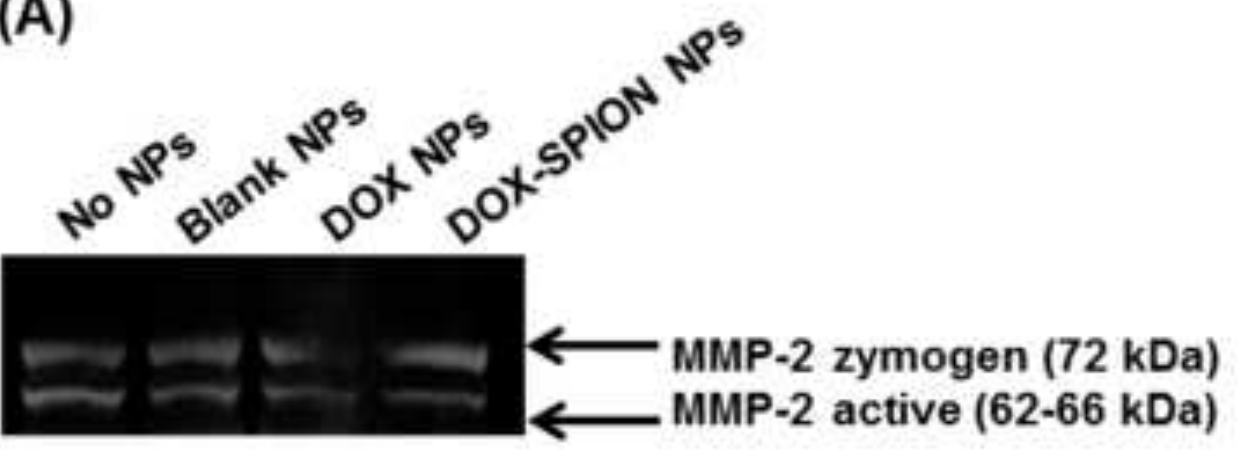

[0.
(B)

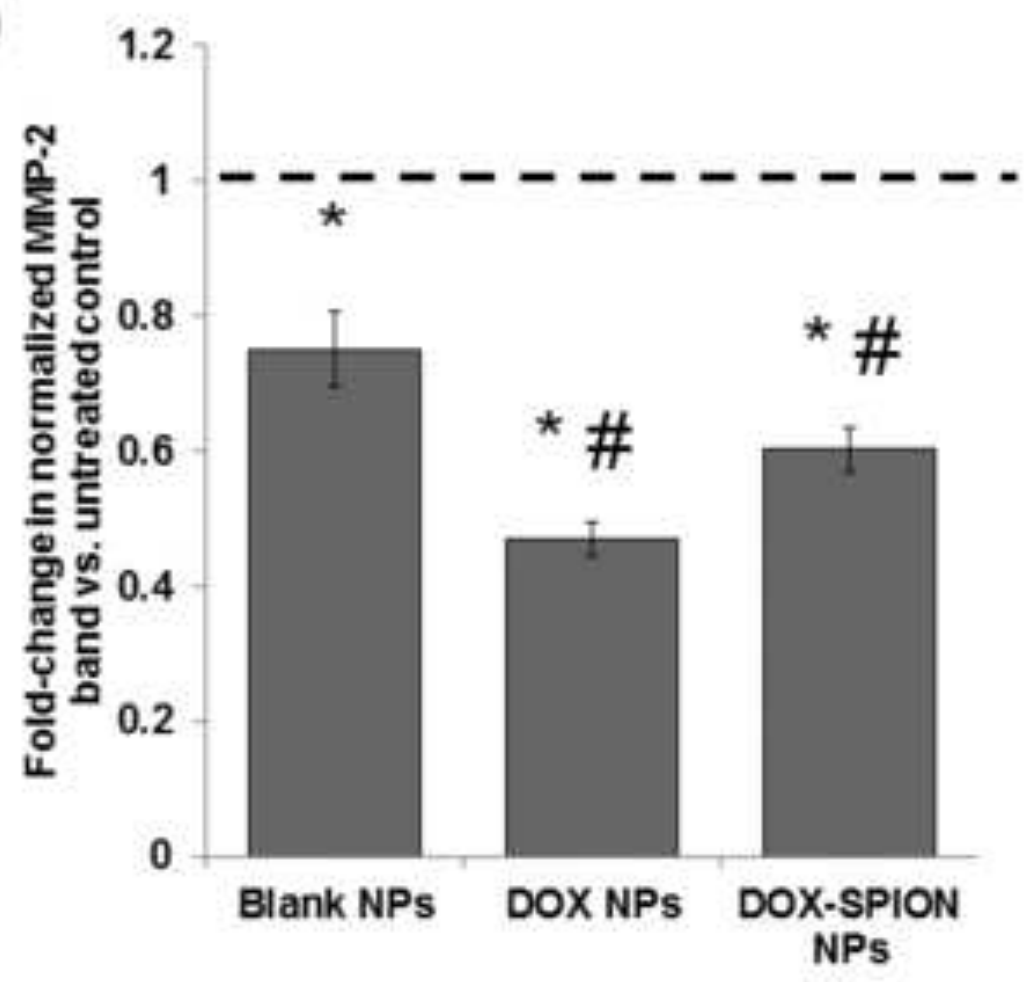

Figure 7 
(A)

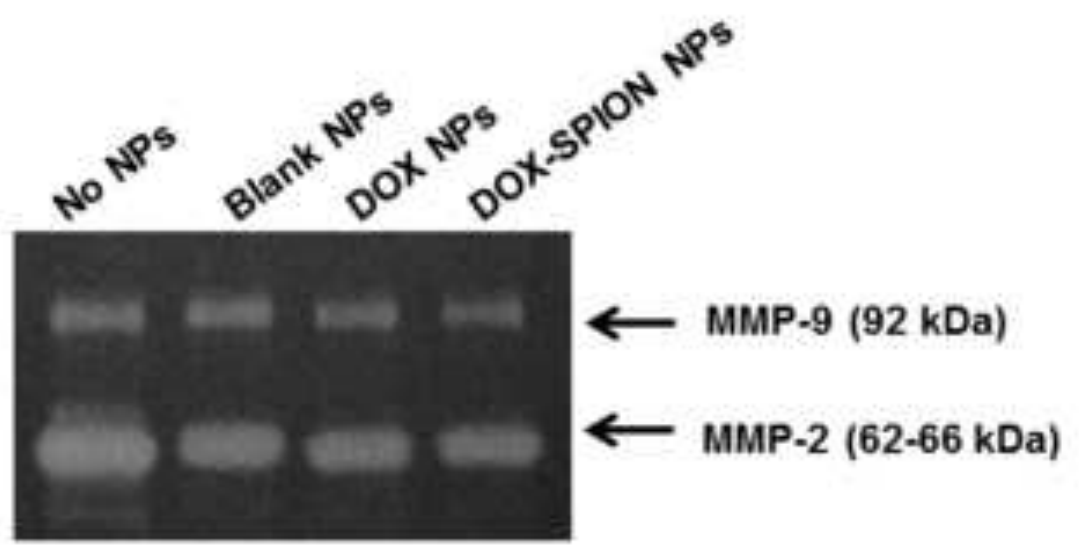

Figure 8
(B)

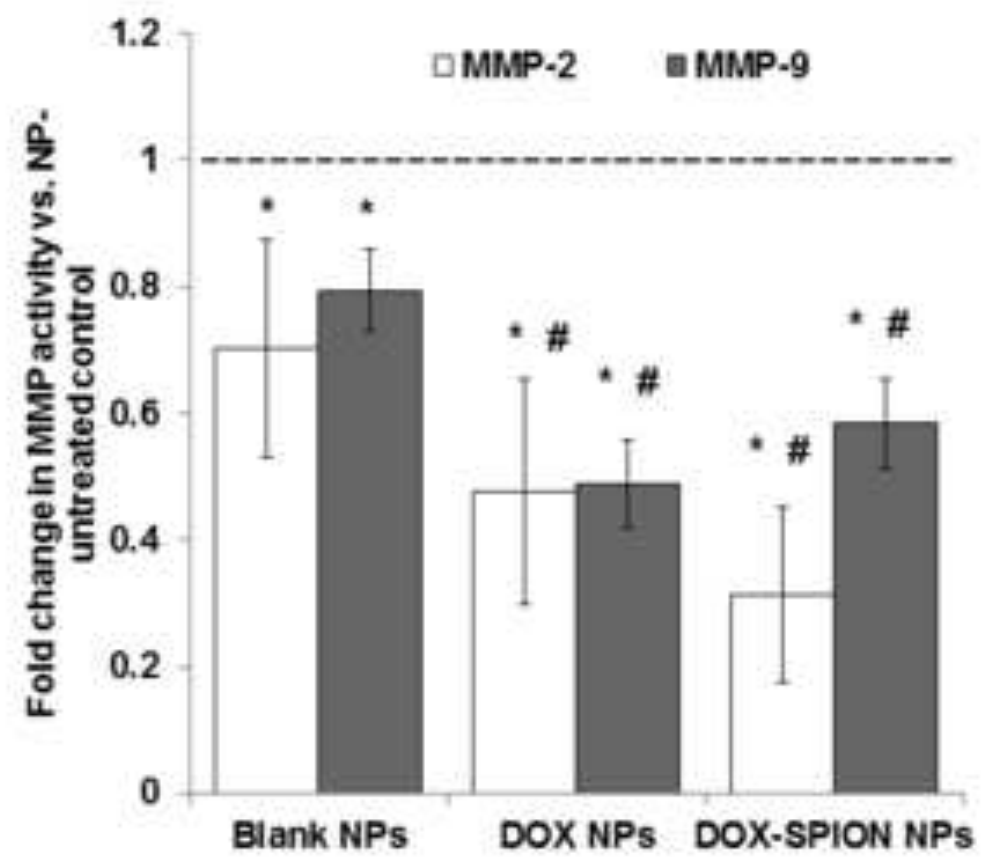


IF Control
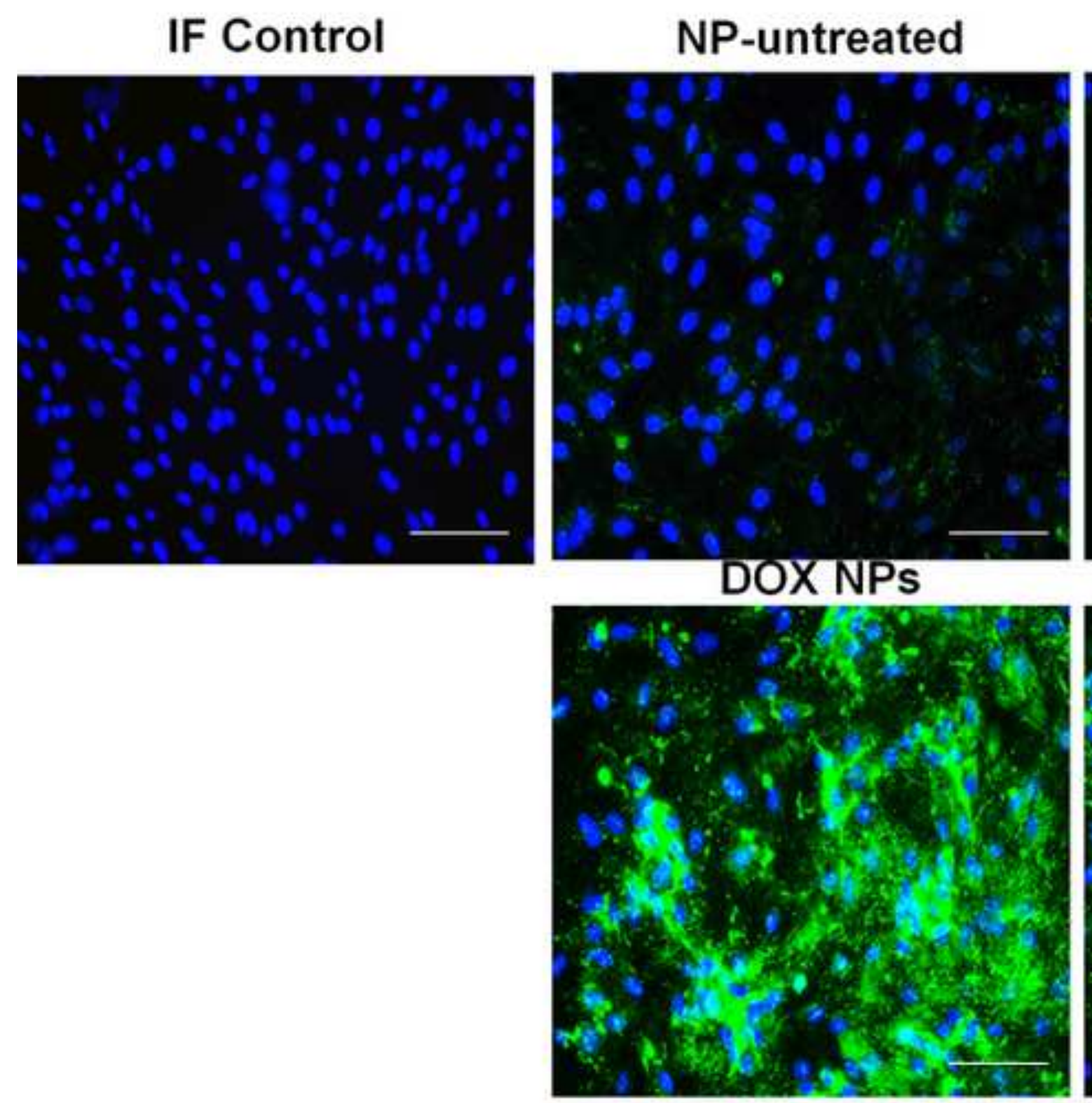

Figure 9

NP-untreated
Blank NPs

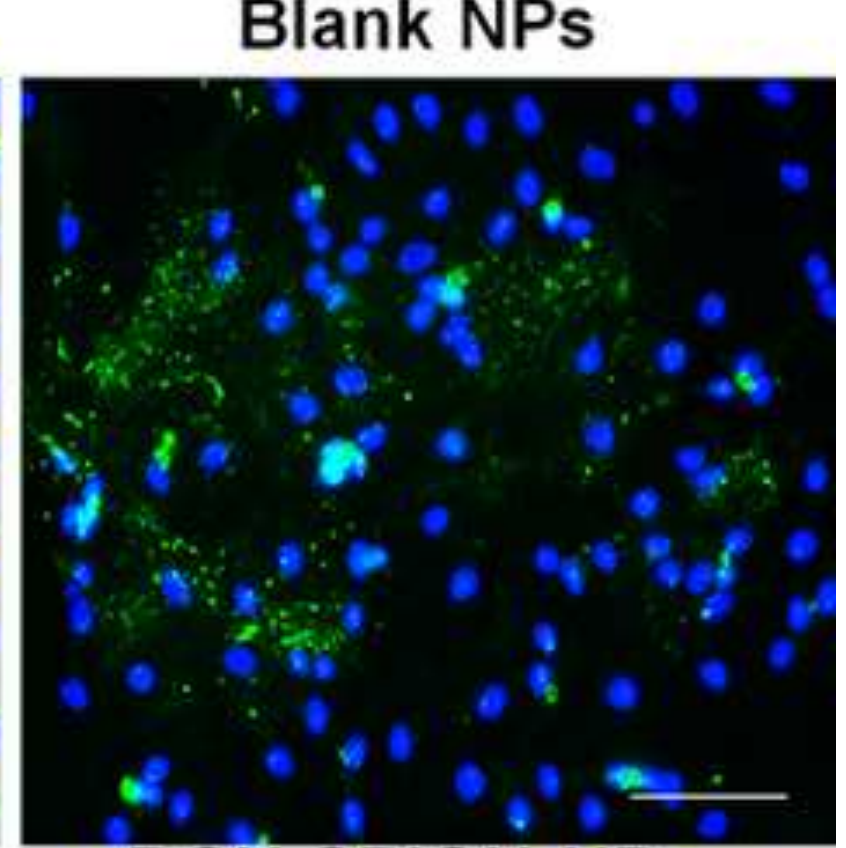

DOX-SPION NPS

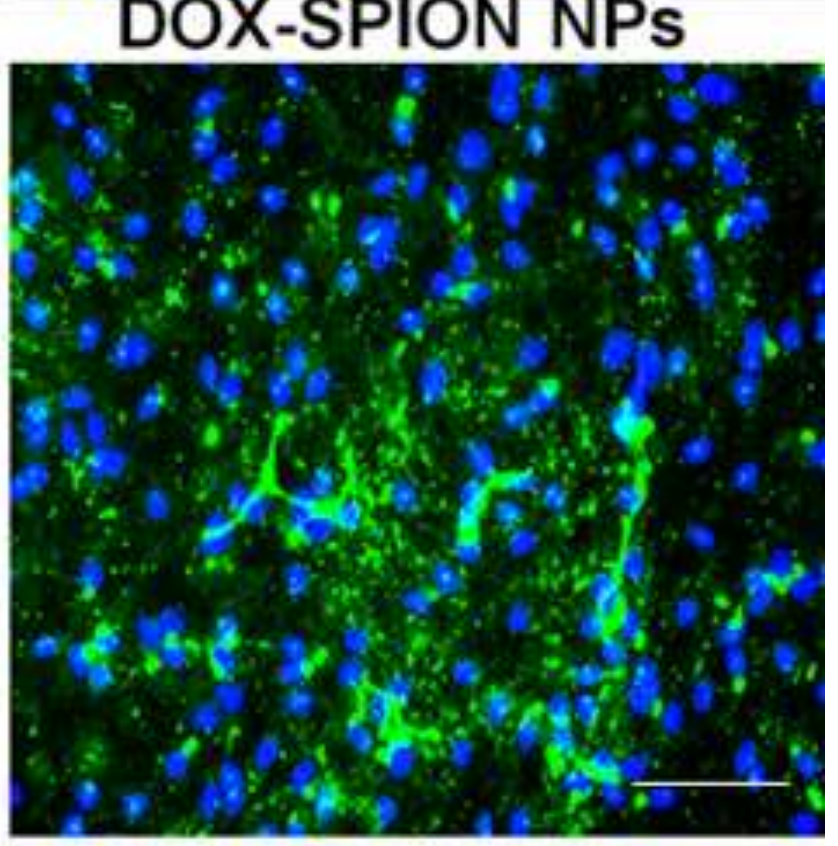

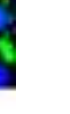



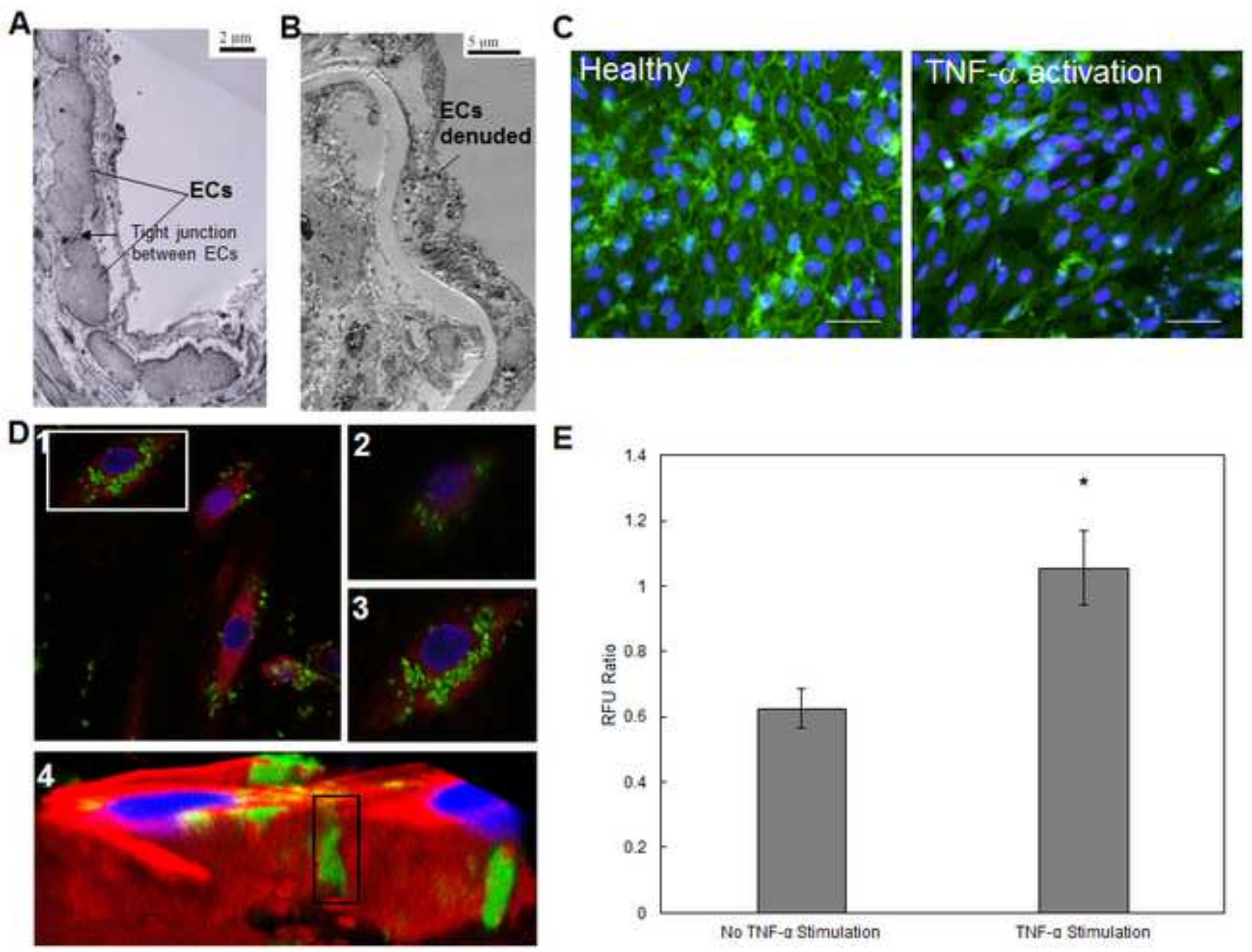

Figure 10 
(A)

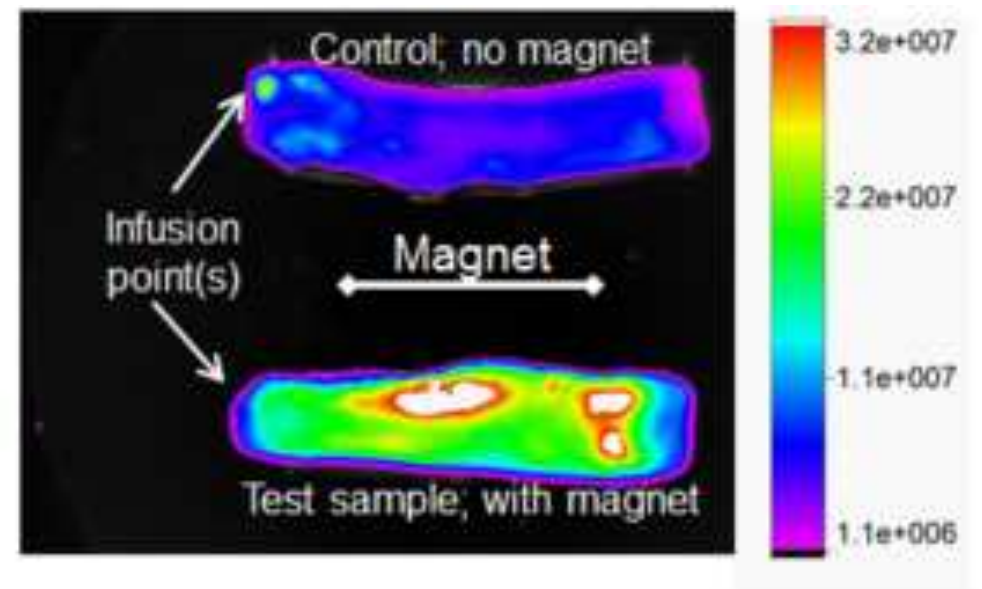

(B)

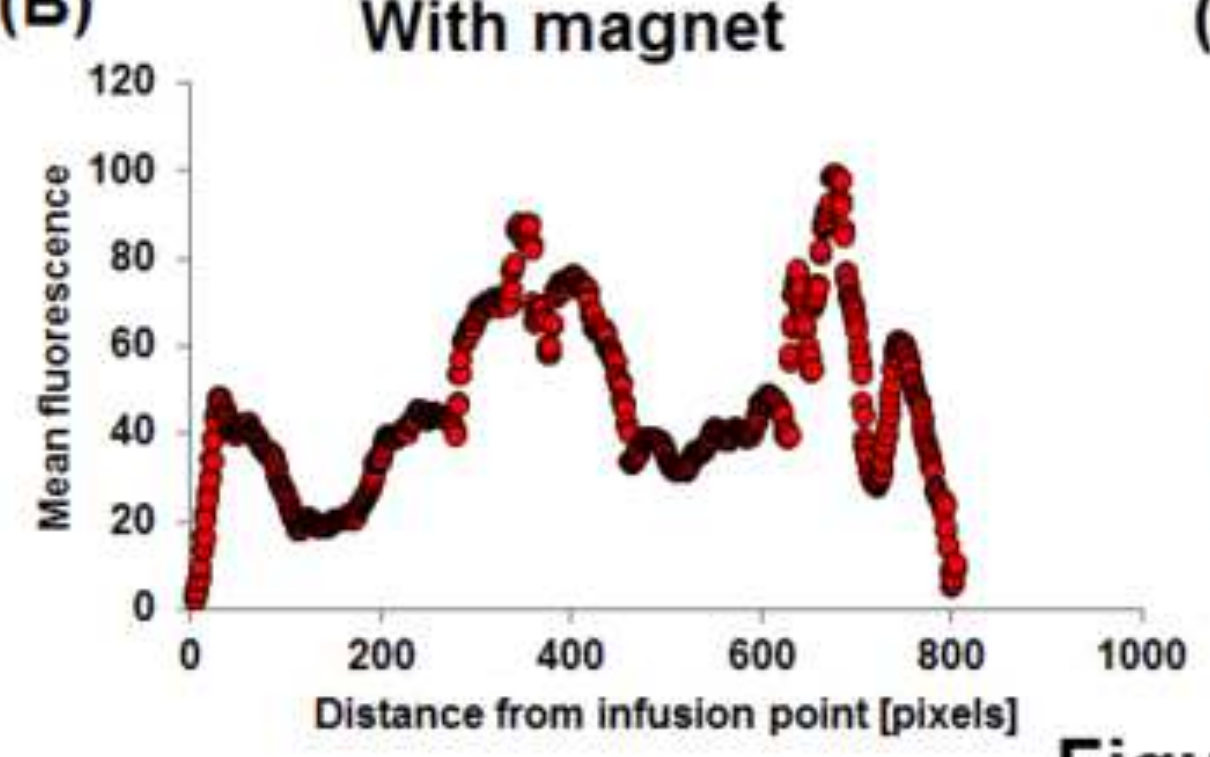

(C)

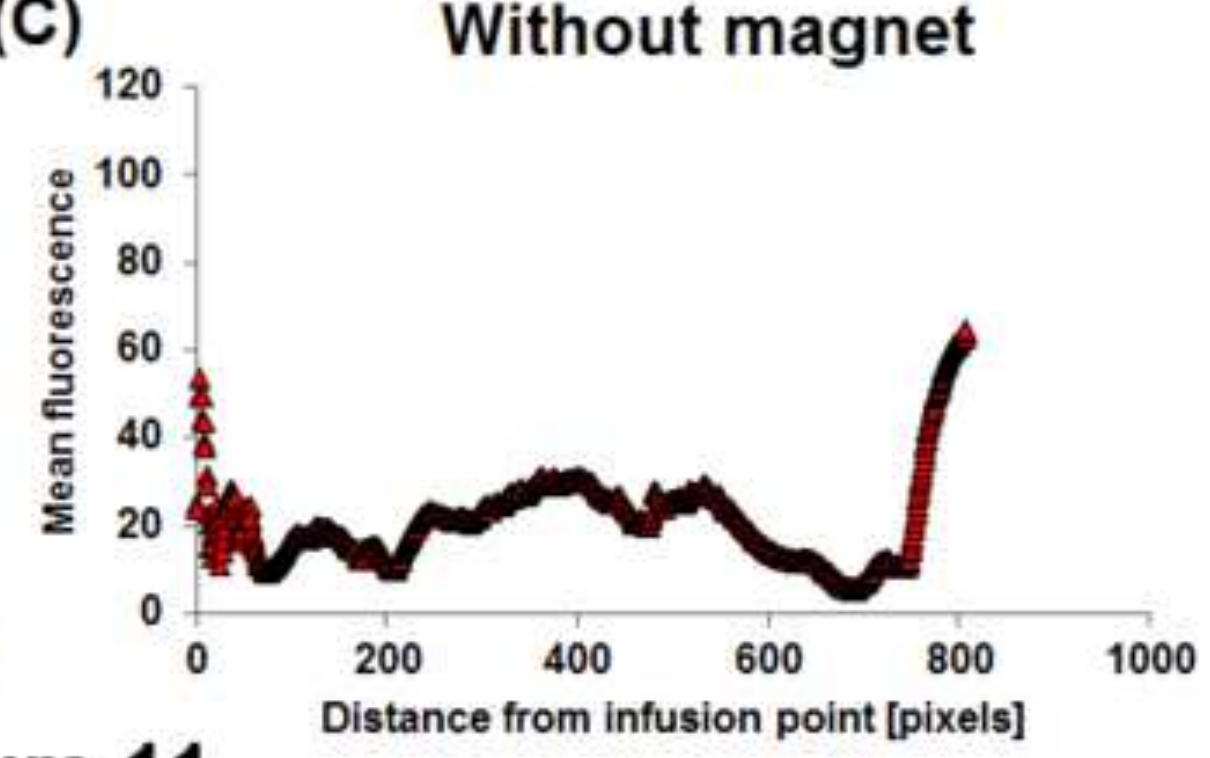

Fiqure 11 
Mode of Delivery of Matrix-

Regenerative NPs

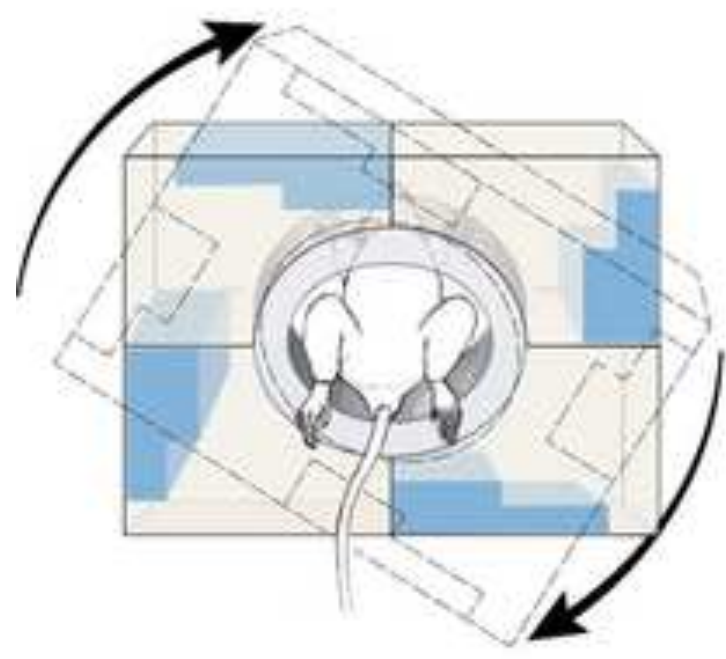

Rotating quadrupolebased permanent magnet

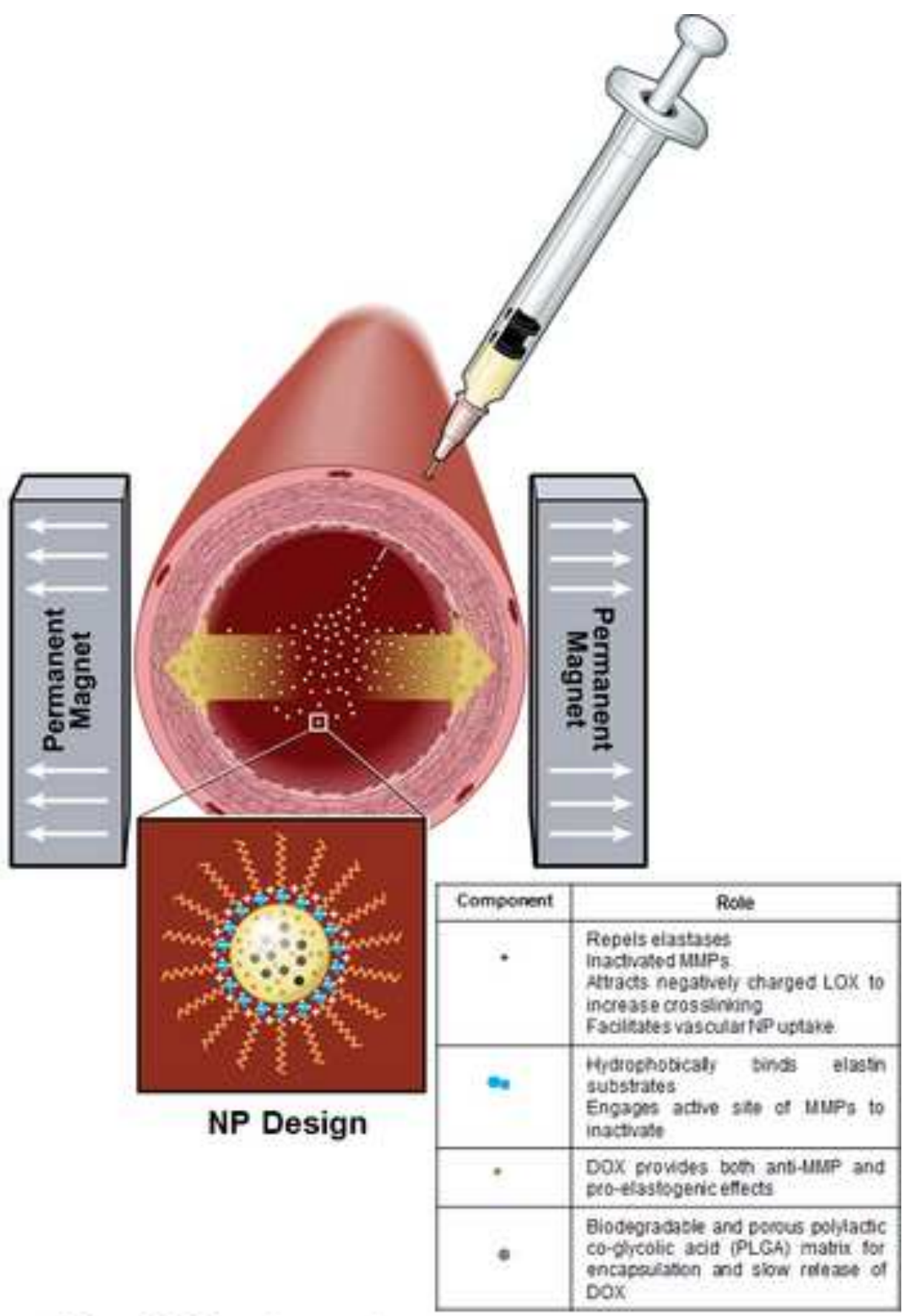

Graphical Abstract 


\section{Statement of Significance}

Proactive screening of high risk elderly patients now enables early detection of abdominal aortic aneurysms (AAAs). There are no established drug-based therapeutic alternatives to surgery for AAAs, which is unsuitable for many elderly patients, and none which can achieve restore disrupted and lost elastic matrix in the AAA wall, which is essential to achieve growth arrest or regression. We have developed a first generation design of polymer nanoparticles (NPs) for AAA tissue localized delivery of doxycycline, a modified tetracycline drug at low micromolar doses at which it provides both pro-elastogenic and anti-proteolytic benefits that can augment elastic matrix regenerative repair. The nanocarriers themselves are also uniquely chemically functionalized on their surface to also provide them pro-elastin-regenerative $\&$ anti-matrix degradative properties. To provide an active driving force for efficient uptake of intra-lumenally infused NPs to the AAA wall, in this work, we have rendered our polymer NPs mobile in an applied magnetic field via co-incorporation of super-paramagnetic iron oxide NPs. We demonstrate that such modifications significantly improve wall uptake of the NPs with no significant changes to their physical properties and regenerative benefits. Such NPs can potentially stimulate structural repair in the AAA wall following one time infusion to delay or prevent AAA growth to rupture. The therapy can provide a non-surgical treatment option for high risk AAA patients. 